Pacific Journal of Mathematics

GALOIS THEORY FOR BANACH ALGEBRAS 


\title{
GALOIS THEORY FOR BANACH ALGEBRAS
}

\author{
DAVID T. BRowN
}

This paper deals with the classical Galois theory in the context of the Arens-Hoffman extension $B=A[x] /(\alpha(x))$ of a commutative Banach algebra $A$ (with identity over the complex field $\mathscr{C}$ ) with respect to a monic polynomial $\alpha(x)$ over $A$ with an invertible discriminant. We show that the fundamental theorem of the Galois theory for commutative rings [S.U. Chase, D.K. Harrison, and A. Rosenberg, Galois theory and cohomology of commutative rings, Memoirs, Amer. Math. Soc. No. 52 (1965)] applies to our situation. The fixed algebras of the subgroups of the Galois group are then characterized for the case where $A$ is semi-simple. The techniques are primarily topological and consist in examining the relationships between $\Phi_{B}$ and $\Phi_{A}$, where the $\Phi^{\prime}$ 's denote the respective carrier spaces of the Banach algebras $A$ and $B$ together with the usual weak * topology.

The topological techniques referred to in the abstract, stem from two results. The first is by J.A. Lindberg, Jr. [12, Proposition 1.3] which shows that $\Phi_{B}$ possesses a property similar to that possessed by a covering space of $\Phi_{A}$. The other result is by G.A. Heuer [6, Th. 3.5] which shows that under certain connectedness assumptions, $\Phi_{B}$ is a covering space (in the sense of Chevalley [5]) of $\Phi_{A}$. This classical notion of a covering space will not be used since we do not wish to limit ourselves to working with a connected and locally connected space $\Phi_{A}$. At the other extreme, we do not need the full generality used by S. Lubkin in [13]. We will therefore modify Lubkin's definition and work with our own version of a covering space. The first section of this paper develops all aspects of this notion which we will be using.

We present some of the basic notions of the Arens-Hoffman extension in $\S 2$ and then show that in a very special case (which includes the case where $\Phi_{A}$ is a connected space), the fundamental theorem of the Galois theory for commutative rings [4] can be applied.

In $\S 3$ and $\S 4$, we characterize the fixed algebras of the subgroups of the Galois group in terms of our notion of a covering space. This will be done under the assumptions that $A$ is semi-simple and that the generating polynomial $\alpha(x)$ factors completely over $B$. After obtaining results for the special case dealt with in $\S 2$, we prove corresponding results for the general situation.

This paper builds upon the work of G.A. Heuer [6], J.A. Lindberg, Jr. [11] and [12] and Heuer and Lindberg [7]. 
1. Definition. Let $X$ and $Y$ be topological spaces, and let $p$ be a continuous mapping of $X$ onto $Y$. We will say that $(X, p)$ is a local covering space of $Y$ if card $\left(p^{-1}(y)\right)$ (= cardinality of the set $\left.\left\{p^{-1}(y)\right\}\right)$ is a finite constant for each $y \in Y$ (this constant may depend on $y \in Y$ ) and if for each $y \in Y$ with $p^{-1}(y)=\left\{x_{1}, x_{2}, \cdots, x_{k}\right\}$, there exists disjoint neighborhoods $U_{1}, U_{2}, \cdots, U_{k}$ in $X$ of $x_{1}, x_{2}, \cdots, x_{k}$ respectively such that $p$ restricted to each $U_{i}$ is a homeomorphism of $U_{i}$ onto $p\left(U_{1}\right)$ and $p^{-1}\left(p\left(U_{1}\right)\right)=\bigcup_{i=1}^{k} U_{i}$.

In the event $(X, p)$ is a local covering space of $Y$ with the property that card $\left(p^{-1}(y)\right)$ is a constant independent of $y \in Y$, we will say that $(X, p)$ is a covering space of $Y$.

We give a necessary and sufficient condition that $(X, p)$ be a local covering space of $Y$ in the case where both $X$ and $Y$ are compact Hausdorff spaces. We note that the compactness of $X$ implies that the cardinality of each fiber $p^{-1}(y)$ in $X$ must not only be finite, but must also be bounded by some integer.

THEOREM 1.1. Assume $X$ and $Y$ are compact Hausdorff spaces and $p$ is a continuous mapping of $X$ onto $Y$. Then $(X, p)$ is a local covering space (covering space) of $Y$ if and only if $p$ is an open mapping with the property that for each $x \in X$, there exists a neighborhood $V_{x}$ in $X$ of $x$ such that $p$ restricted to $V_{x}$ is one-to-one (and card $\left(p^{-1}(y)\right)$ is a finite constant independent of $\left.y \in Y\right)$.

Proof. Assume $(X, p)$ is a local covering space of $Y$. Let $V$ be an open subset in $X$ and select any point $y_{0} \in p(V)$ with $p^{-1}\left(y_{0}\right)=$ $\left\{x_{1}, \cdots, x_{k}\right\}$. Let $U_{1}, U_{2}, \cdots, U_{k}$ be disjoint open neighborhoods in $X$ of $x_{1}, x_{2}, \cdots, x_{k}$ respectively such that $U_{1} \cap V \neq \phi, p$ restricted to each $U_{i}$ is a homeomorphism of $U_{i}$ onto $p\left(U_{1}\right)$ and $p^{-1}\left(p\left(U_{1}\right)\right)=\mathbf{U}_{i=1}^{k} U_{i}$.

For each $i$, set $V_{i}=U_{i} \cap p^{-1}\left(p\left(U_{1} \cap V\right)\right)$. It follows that the $V_{i}$ 's are mutually disjoint, $p\left(V_{i}\right)=p\left(U_{1} \cap V\right)$ for each $i$ and $p^{-1}\left(p\left(V_{1}\right)\right)=$ $\mathrm{U}_{i=1}^{k} V_{i}$. Since $p$ restricted to each $U_{i}$ is a homeomorphism, each $V_{i}$ is open with respect to the relative topology of $U_{i}$. Since the latter set is open, each $V_{i}$ is open in $X$.

Since $X-p^{-1}\left(p\left(V_{1}\right)\right)$ is a compact subset of $X, p\left(X-p^{-1}\left(p\left(V_{1}\right)\right)\right)=$ $Y-p\left(V_{1}\right)$ is a compact, and therefore closed, subset of $Y$. Therefore $p\left(V_{1}\right)=p\left(U_{1} \cap V\right)$ is an open subset of $Y$ which contains $y_{0}$ and which is contained in $p(V)$. Thus, $p(V)$ is an open subset of $Y$. Consequently, $p$ is an open mapping.

To prove the converse, assume $p$ is an open mapping, which satisfies the given condition. Let $y_{0} \in Y$ and set $p^{-1}\left(y_{0}\right)=\left\{x_{1}, \cdots, x_{k}\right\}$. Since $X$ is a Hausdorff space, there exist mutually disjoint open neighborhoods $V_{1}, V_{2}, \cdots, V_{k}$ in $X$ of $x_{1}, x_{2}, \cdots, x_{k}$ respectively. We assume that $p$ restricted to each $V_{i}$ is one-to-one (if not, replace each $V_{i}$ by $V_{i} \cap$ 
(interior of $\left.V_{x_{i}}\right)$ ). Let $U_{0}=\bigcap_{i=1}^{k} p\left(V_{i}\right)$ and let $U_{i}=V_{i} \cap p^{-1}\left(U_{0}\right)$ for each $i=1,2, \cdots, k$. Thus $\bigcup_{i=1}^{k} U_{i} \subset p^{-1}\left(U_{0}\right)$. Since $p$ is a continuous open mapping, $U_{0}$ is an open neighborhood in $Y$ of $y_{0}$ and each $U_{i}$ is an open neighborhood in $X$ of $x_{i}$. The $U_{i}$ 's are mutually disjoint and each of them is mapped onto $U_{0}$ by $p$.

Now let $W=U_{0} \cap p\left(X-\bigcup_{i=1}^{k} U_{i}\right)$. This subset of $Y$ is closed with respect to the relative topology of $U_{0}$. If $W$ is the empty set, then $p^{-1}\left(U_{0}\right) \subset \bigcup_{i=1}^{k} U_{i}$ and therefore $p^{-1}\left(U_{0}\right)=\bigcup_{i=1}^{k} U_{i}$. Also $p \mid U_{i}$ ( $p$ restricted to $U_{i}$ ) is one-to-one for $i=1,2, \cdots, k$. Finally $p \mid U_{i}$ is a homeomorphism since each $U_{i}$ is open and $p$ is an open mapping. This completes the proof for the case $W=\phi$. If, on the other hand, there is a point $y \in Y$ such that $y \in W$, then there is a set $W_{0}$ in $Y$ which is open with respect to the relative topology of $U_{0}$ and which contains $y_{0}$ but is disjoint from $W$. This means that $p^{-1}\left(W_{0}\right) \subset \bigcup_{i=1}^{k} U_{\imath}$. Since $U_{0}$ is open in $Y$ and since $y_{0} \in W_{0}, W_{0}$ is an open neighborhood in $Y$ of $y_{0}$. For each $i$, let $W_{i}=U_{i} \cap p^{-1}\left(W_{0}\right)$. This set is an open set in $X$ which contains $x_{i}$. It follows that $p\left(W_{i}\right)=W_{0}$ for $i=$ $1,2, \cdots, k$ and $p^{-1}\left(W_{0}\right)=\bigcup_{i=1}^{k} W_{i}$. The proof is now completed by applying to the $W_{i}^{\prime}$ 's the argument used on the $U_{i}^{\prime}$ 's in the case $W=\phi$.

From now on, when we write the phrase " $(V, r)$ is a local covering space (or covering space) of $U$ ', we will assume both $V$ and $U$ are compact Hausdorff spaces and $r$ is a continuous mapping of $V$ onto $U$. Note that a subset of a compact Hausdorff space is closed if and only if it is compact, and a continuous mapping between two such spaces is a closed mapping. Also, if $r$ and $s$ are two mappings such that the composite mapping $r \circ s$ is defined, we will write $r s$ for $r \circ s$.

Lemma 1.2. Let $(X, p)$ and $(Z, q)$ be two local covering spaces of $Y$ and assume $w$ is a continuous mapping of $X$ into $Z$ such that $p=q w$. Then $w$ is an open mapping.

Proof. Let $U$ be an open subset of $X$ and suppose $z \in w(U)$. Select a point $x \in w^{-1}(z) \cap U$. Since $(Z, q)$ is a local covering space of $Y$, there exists an open neighborhood $V$ in $Z$ of $z$ such that $q \mid V$ is one-to-one. We assume $V$ is so small that $q(V) \subset p(U)$. There also exists an open neighborhood $U_{0}$ in $X$ of $x$ such that $U_{0} \subset w^{-1}(V) \cap U$. By replacing $U_{0}$ by $U_{0} \cap p^{-1}\left(p\left(U_{0}\right) \cap q(V)\right)$ and $V$ by $U_{0} \cap q^{-1}\left(p\left(U_{0}\right) \cap q(V)\right)$ if need be, we may and do assume that $p\left(U_{0}\right)=q(V)$.

Now let $z_{0} \in V$. There is a point $x_{0} \in U_{0}$ such that $p\left(x_{0}\right)=q\left(z_{0}\right)$. Therefore $q w\left(x_{0}\right)=p\left(x_{0}\right)=q\left(z_{0}\right)$. Since $U_{0} \subset w^{-1}(V), w\left(x_{0}\right) \in V$. But since $q \mid V$ is one-to-one, $w\left(x_{0}\right)=z_{0}$. This means $z_{0} \in w\left(U_{0}\right)$. Thus $V \subset w\left(U_{0}\right) \subset w(U)$. The fact that $z \in V$ and $V$ is open implies that $w(U)$ is an open subset of $Z$. This completes the proof. 
If $(X, p)$ is a local covering space of $Y$, denote by $E(X: Y)$ the group of all homeomorphisms $\phi$ of $X$ onto itself with the property that $p \phi=p$.

Lemma 1.3. Assume $(X, p)$ and $(Z, q)$ are two local covering spaces of $Y$. Suppose $w$ and $u$ are two continuous mappings of $X$ into $Z$ such that $p=q w=q u$. Then $H=\{x \in X: w \phi(x)=u(x)$ for any $\phi \in E(X: Y)\}$ is an open and closed subset of $X$.

Proof. Since $w \phi$ and $u$ are both continuous mappings of $X$ into the Hausdorff space $Z, H$ is closed in $X$. (See, for example, [10, Problem C, p. 100]).

To show $H$ is also open, let $x_{0} \in H$ and set $z_{0}=u\left(x_{0}\right)=w \phi\left(x_{0}\right)$. Let $U$ be any open neighborhood in $Z$ of $z_{0}$ such that $q \mid U$ is one-toone and let $V$ be any open neighborhood in $X$ of $x_{0}$ such that $u(V) \cap w \phi(V) \subset U$. If $x \in V$, then $u(x)$ and $w \phi(x)$ are both in $U$, and furthermore, $q u(x)=p(x)=p \dot{\phi}(x)=q w \phi(x)$. Since $q \mid V$ is one-to-one, $u(x)=w \dot{\phi}(x)$. This means $x \in H$. We have thus shown that $V \subset H$ which implies that $H$ is an open subset. This completes the proof.

The next result enables us to work first with components of spaces and then to extend to open and closed subsets. An equivalent form of the following lemma can be found in [8, p. 47].

Lemma 1.4. Let $C$ be a component in a compact Hausdorff space $X$ and let $U$ be any open set in $X$ which contains $C$. Then there exists an open and closed set $K$ in $X$ which contains $C$ and which is contained in $U$.

Lemma 1.5. Assume $(X, p)$ is a local covering space of $Y$ and let $K$ be an open and closed subset of $X$. Then for any integer $s$, $K_{s}=\left\{y \in Y: \operatorname{card}\left(p^{-1}(y) \cap K\right)=s\right\}$ is an open and closed subset of $Y$.

Proof. Let $y \in K_{s}$ and let $p^{-1}(y) \cap K=\left\{x_{1}, x_{2}, \cdots, x_{s}\right\}$. Select open neighborhoods $V_{i}$ in $X$ of $x_{i}$ for $i=1,2, \cdots, s$ such that (i) each $V_{i}$ is contained in $K$ (ii) $p$ restricted to each $V_{i}$ is a homeomorphism of $V_{i}$ onto a set $V_{0}$ in $Y$, and (iii) $p^{-1}\left(V_{0}\right) \cap K=\bigcup_{i=1}^{s} V_{i}$. This choice of the $V$ 's is possible since $p$ is an open mapping. (The details are essentially the same as in the proof of Theorem 1.1).

$V_{0}$ is an open neighborhood in $Y$ of $y$. It follows from (ii) and (iii) that $V_{0}$ is contained in $K_{s}$. This means $K_{s}$ is an open set. But $K_{s}$ must also be closed since $p(K)$ is the finite union of $K_{i}(i=1,2$, $\cdots, N$ ) and the $K_{i}$ 's are mutually disjoint. (Recall the comment prior to Theorem 1.1 that there exists an integer $N$ such that card $\left(p^{-1}(y)\right) \leqq N$ for all $y \in Y)$. 
Our main interest in local covering spaces is to obtain properties of the group $E(X: Y)$ of "covering homeomorphisms". The next result is the first of our two main technical lemmas which deal with this situation.

LEMma 1.6. Assume $(X, p)$ is a local covering space of $Y$ and assume $K$ is an open and closed subset of $X$. Also suppose that $x_{0}$ and $x_{0}^{\prime}$ are two distinct points of $K$, and that $r$ and $s$ are two continuous mappings of $X$ into itself such that $p r=p=p s, r\left(x_{0}\right)=x_{0}^{\prime}$, and $s\left(x_{0}^{\prime}\right)=x_{0}$. Then there is a homeomorphism $\phi$ in $E(X: Y)$ such that $\phi\left(x_{0}\right)=x_{0}^{\prime}, \phi(K)=K$, and $\phi$ is the identity homeomorphism on $X-K$.

Proof. Let $H=\{x \in X: s r(x)=x\}$. This is a nonempty subset of $X$ which, by Lemma 1.3 is open and closed. Since $s r \mid H$ is the identity mapping, $r \mid H$ is one-to-one. Both mappings are closed since $X$ is a compact Hausdorff space. Moreover by Lemma 1.2, both mappings are also open.

Let $C$ and $D$ be the components in $X$ such that $x_{0} \in C$ and $x_{0}^{\prime} \in D$. Then $C \subset K \cap H, D \subset K, r(C) \subset D$ and $s(D) \subset C$. Therefore, $C=$ $s r(C) \subset s(D) \subset C$. Consequently, $s(D)=C$. Furthermore $r(C)=D$ since $D \subset r(H)$ and $r s \mid r(H)$ is the identity mapping. If $C$ and $D$ are distinct components, then there exists disjoint open sets $U$ and $V$ in $X$ such that $C \subset U \subset K \cap H, D \subset V \subset K \cap r(H)$, and $r(U) \subset V$. By Lemma 1.4, there exists an open and closed set $K_{0}$ in $X$ such that $C \subset K_{0} \subset U$. Thus $r\left(K_{0}\right) \subset r(U) \subset V$. If follows that the sets $r\left(K_{0}\right)$ and $K_{0}$ are disjoint. Define a mapping $\phi$ of $X$ into itself as follows:

$$
\dot{\rho}\left|K_{0}=r\right| K_{0}, \quad \dot{\phi}\left|r\left(K_{0}\right)=s\right| r\left(K_{0}\right),
$$

and $\phi$ is the identity mapping on $X-K_{0} \cup r\left(K_{0}\right)$. This is a one-to-one mapping of $K_{0}$ onto $r\left(K_{0}\right)$ and of $r\left(K_{0}\right)$ on $K_{0}$. Since $K_{0}$ and $r\left(K_{0}\right)$ are disjoint, $\phi \in E(X: Y)$. Also, $\dot{\phi}\left(x_{0}\right)=x_{0}^{\prime}$, and the fact that $K_{0} \subset K \mathrm{im}$ plies $\phi(K)=K$ and $\phi$ is the identity off of $K$. This completes the proof for the case where $C$ and $D$ are distinct components.

On the other hand, if $C$ and $D$ are the same component, then $r(C)=C=s(C)$. Since $p(C)$ is a closed and connected subset of $Y$, the argument used in the proof of [12, Th. 2.4] shows that $p^{-1}(p(C))$ is a finite union of disjoint connected sets each of which is open (and therefore closed) in the relative topology of $p^{-1}(p(C))$. This means that there exists an open set $U$ in $X$ such that $U \cap p^{-1}(p(C))=C$. We assume, without loss of generality, that $U$ is a subset of $H \cap r(H) \cap K$. By Lemma 1.4, there exists an open and closed subset $K_{0}$ in $X$ such that $C \subset K_{0} \subset U$. It follows that $K_{0} \cap p^{-1}(p(C))=C$. Also, $K_{0} \subset H \cap r(H)$ implies that both $r \mid K_{0}$ and $s \mid K_{0}$ are one-to-one mappings.

Let $y_{1}$ be any element of $p(C)$ and assume card $\left(p^{-1}\left(y_{1}\right) \cap C\right)=m$. 
It follows from Lemma 1.5 that $K_{m}=\left\{y \in p\left(K_{0}\right)\right.$ : $\left.\operatorname{card}\left(p^{-1}(y) \cap K_{0}\right)=m\right\}$ is an open and closed subset of $Y$. Since $p(C)$ is connected and has a nonempty intersection with $K_{m}$, it must be a subset of $K_{m}$. Therefore, for any $y \in p(C)$, card $\left(p^{-1}(y) \cap C\right)=\operatorname{card}\left(p^{-1}(y) \cap p^{-1}(p(C)) \cap K_{0}\right)=$ card $\left(p^{-1}(y) \cap K_{0}\right)=m$.

Now let $P=\left\{y \in K_{m}: r\left(p^{-1}(y) \cap K_{0}\right) \subset K_{0}\right\} . \quad P$ is a subset of $Y$ which contains $p(C)$ and is contained in $K_{m}$. We note that $y \in P$ if and only if $y \in K_{m}$ and $r$ maps $p^{-1}(y) \cap K_{0}$ onto itself. We will show that $P$ is an open and closed subset of $Y$.

(i) $P$ is an open set. Let $y_{0} \in P$ and assume $p^{-1}\left(y_{0}\right) \cap K_{0}=$ $\left\{x_{0}^{1}, x_{0}^{2}, \cdots, x_{0}^{m}\right\}$. For each $i=1,2, \cdots, m$, let $U_{i}$ be an open neighborhood in $X$ of $x_{0}^{i}$ such that both $U_{i}$ and $r\left(U_{i}\right)$ are contained in the open and closed set $p^{-1}\left(K_{m}\right) \cap K_{0}$ and such that $p$ restricted to each $U_{i}$ is a homeomorphism of $U_{i}$ onto a set $U_{0}$ in $Y$. For any point $y \in U_{0}, p^{-1}(y) \cap K_{0} \subset p^{-1}\left(K_{m}\right) \cap K_{0}$. This means card $\left(p^{-1}(y) \cap K_{0}\right)=m$. Consequently, $p^{-1}(y) \cap K_{0} \subset \bigcup_{i=1}^{m} U_{\imath}$. Thus for $x \in p^{-1}(y) \cap K_{0}, r(x)$ is an element of $r\left(U_{j}\right)$ for some $j$. Since the latter set is a subset of $K_{0}, r\left(p^{-1}(y) \cap K_{0}\right) \subset p^{-1}(y) \cap K_{0}$. Therefore $U_{0} \subset P$. It follows, since $U_{0}$ is open, that $P$ is an open set.

(ii) $P$ is a closed set. Let $y_{1}$ be a point in the closure of $P$ and assume there exists $x_{1} \in p^{-1}\left(y_{1}\right) \cap K_{0}$ such that $r\left(x_{1}\right) \notin K_{0}$. (i.e. assume $y_{1} \notin P$.) Let $\mathrm{V}$ be any open neighborhood in $X$ of $x_{1}$ such that $V \subset p^{-1}\left(K_{m}\right) \cap V_{0}$ and $r(V) \cap K_{0}=\dot{\phi}$. Since $p(V)$ is an open neighborhood in $Y$ of $y_{1}$, there exists a point $y$ in $p(V) \cap P$. But for any $x$ in $p^{-1}(y) \cap V, r(x)$ must be a point in $p^{-1}(y) \cap K_{0}$. Thus $r(V) \cap K_{0}$ is nonempty. This is a contradiction. Therefore any $x_{1}$ in $p^{-1}\left(y_{1}\right) \cap K_{0}$ has the property that $r\left(x_{1}\right) \in K_{0}$. This means $y_{1} \in P$ and thus $P$ is a closed set.

Finally, consider the set $Q=p^{-1}(P) \cap K_{0}$. This is an open and closed set in $X$ which contains $C$. Since $p r=p$, and $r\left(p^{-1}(P)\right) \subset p^{-1}(P)$, $r$ maps $Q$ into itself. But the fact that $r \mid K_{0}$ is one-to-one and card $\left(p^{-1}(y) \cap K_{0}\right)=m$ for all $y \in P$ implies that $r$ must map $Q$ onto itself.

Define a mapping $\phi$ of $X$ into itself by $\phi|Q=r| Q$ and $\phi$ is the identity elsewhere. Since $K_{0} \subset K$ and $r \mid K_{0}$ is one-to-one, $\phi \mid K$ is a one-to-one mapping of $K$ onto itself. It follows that $\phi\left(x_{0}\right)=x_{0}^{\prime}$ and $\phi \in E(X: Y)$. This completes the proof for the case where $C$ and $D$ are the same component. Therefore the proof of Lemma 1.6 is complete.

We have the following corollary to the proof of the above lemma.

Corollary 1.7. Assume $(X, p)$ is a local covering space of $Y$ and let $C$ be a component of $X$. Then card $\left(p^{-1}(y) \cap C\right)$ is a constant, 
say $m$, independent of $y \in p(C)$. Furthermore, if $K$ is any open and closed set in $X$ which contains $C$ and if $r$ is a continuous mapping of $X$ into itself such that $p r=p$ and $r(C)=C$, then there exists an open and closed set $Q$ in $X$ with the following properties: (i) $C \subset Q \subset K$, (ii) $r(Q)=Q$, (iii) card $\left(p^{-1}(y) \cap Q\right)=m$ for all $y \in p(Q)$, and (iv) $p^{-1}(p(C)) \cap Q=C$.

We also remark that if $C$ and $D$ are distinct components such that $p(C)=p(D)$, then the corresponding open and closed sets $Q_{C}$ and $Q_{D}$ given by the above corollary can be chosen such that they are disjoint and such that $p\left(Q_{C}\right)=p\left(Q_{D}\right)$.

The above two results now yield a structure theorem for local covering spaces.

COROLlary 1.8. $(X, p)$ is a local covering space of $Y$ if and only if there exists a finite covering of $Y$ by mutually disjoint, open and closed sets $Y_{1}, \cdots, Y_{k}$ with the property that if $X_{i}=p^{-1}\left(Y_{i}\right)$ and $p_{i}=p \mid X_{i}$, then $\left(X_{i}, p_{i}\right)$ is a covering space of $Y_{i}$ for $i=1,2, \cdots, k$.

Proof. Assume $(X, p)$ is a local covering space of $Y$ and let $R$ be a component of $Y$. Then, as in the second half of the proof of Lemma 1.6, $p^{-1}(R)=\bigcup_{j=1}^{q} R_{j}$, where the $R_{j}$ 's are mutually disjoint, connected, open and closed with respect to the relative topology of $p^{-1}(R)$, and $p\left(R_{j}\right)=p\left(R_{1}\right)$ for each $j=1,2, \cdots, q$. It follows that each $R_{j}$ is a component of $X$. By applying the above corollary to each of the $R_{j}$ 's with $r$ equal to the identity mapping in each case, there exists open and closed sets $Q_{1}, Q_{2}, \cdots, Q_{q}$ in $X$ containing $R_{1}, R_{2}, \cdots$, $R_{q}$ respectively such that if $m_{j}=\operatorname{card}\left(p^{-1}(y) \cap R_{j}\right)$ for each $y \in R$, then $m_{j}=\operatorname{card}\left(p^{-1}(y) \cap Q_{j}\right)$ for each $y \in p\left(Q_{\jmath}\right)$. We assume, without loss of generality, that the $Q_{j}$ 's are mutually disjoint and that $p$ maps each $Q_{j}$ onto a set $Q_{0}$ in $Y$. This latter set is open and closed in $Y$. Thus for each $y \in Q_{0}$, card $\left(p^{-1}(y)\right)=\sum_{j=1}^{q} m_{j}$. This means that $\left(p^{-1}\left(Q_{0}\right)\right.$, $\left.p \mid p^{-1}\left(Q_{0}\right)\right)$ is a covering space of $Q_{0}$. This part of the proof is completed by using the fact that $Y$ is a compact space. Since the converse is immediate, the proof is complete.

Definition. Assume $(X, p)$ is a local covering space of $Y$ and $K$ is any open and closed set in $X$. We will say that a subset $E$ of $E(X: Y)$ is (simply) transitive on the fibers of $K$ if for any $y \in p(K)$ and for any two points $x_{1}$ and $x_{2}$ in $p^{-1}(y) \cap K$, there exists a (unique) homeomorphism $\phi$ in $E$ such that $\phi\left(x_{1}\right)=x_{2}$.

If for any $y \in p(K), \quad$ card $\left(p^{-1}(y) \cap K\right)=m$, then a subset $E$ of $E(X: Y)$ is simply transitive on the fibers of $K$ if and only if $E$ contains exactly $m$ elements no two of which agree at any point of $K$. 
We have an additional corollary to Lemma 1.6.

Corollary 1.9. Assume $(X, p)$ is a local covering space of $Y$ and let $K$ be any open and closed set in $X$. If $E(X: Y)$ is transitive on the fibers of $X$, then $E(K: p(K))$ is transitive on the fibers of $K$.

For the remainder of this section, we will deal exclusively with covering spaces. (Our application of local covering spaces does not occur until the last section of this paper.) The next result is the second of our two major lemmas in this section.

Lemma 1.10. Assume $(X, p)$ is a covering space of $Y$. Then $E(X: Y)$ is transitive on the fibers of $X$ if and only if there exists a subset $E$ of $E(X: Y)$ which is simply transitive on the fibers of $X$.

Proof. Assume there exists elements $\phi_{1}, \phi_{2}, \cdots, \phi_{n}$ in $E(X: Y)$ $\left(n=\operatorname{card}\left(p^{-1}(y)\right)\right.$ for all $\left.y \in Y\right)$ such that for any $x \in X$ and $i \neq j$ $\phi_{i}(x) \neq \phi_{j}(x)$. Let $y \in Y$ and let $x_{1}$ and $x_{2}$ be any two points in $p^{-1}(y)$. Since card $\left(\left\{\phi_{i}\left(x_{1}\right): i=1,2, \cdots, n\right\}\right)=n=\operatorname{card}\left(p^{-1}(y)\right)$, there exists a $j$ such that $\phi_{j}\left(x_{1}\right)=x_{2}$. Thus $E(X: Y)$ is transitive on the fibers of $X$.

Assume $E(X: Y)$ is transitive on the fibers of $X$ and let $D$ be a component in $Y$. We will construct $n$ elements of $E(X: Y)$ no two of which agree at any point of $p^{-1}(D)$. As in the proof of Corollary 1.8, $p^{-1}(D)=\bigcup_{j=1}^{m} X_{j}$ where the $X_{j}$ 's are mutually disjoint components of $X$ which are open and closed with respect to the relative topology of $p^{-1}(D)$. If $X_{i}$ and $X_{j}$ are any two distinct components, then there is at least one homeomorphism $\phi$ in $E(X: Y)$ such that $\phi\left(X_{i}\right) \cap X_{j}$ is nonempty. But the latter set is open and closed with respect to the relative topology of $p^{-1}(D)$. Thus $\phi\left(X_{i}\right)=X_{j}$. Consequently, there is a constant $k$ independent of $j=1,2, \cdots, m$ such that card $\left(p^{-1}(y) \cap X_{j}\right)=$ $k$ for all $y \in D$. (Note that $k m=n)$.

For any $y \in D$, arbitrarily label the points of $p^{-1}(y) \cap X_{j}$ by $x_{1 j}$, $x_{2 j}, \cdots, x_{k j}$. Since $E(X: Y)$ is transitive on the fibers of $X$, for each $i=1,2, \cdots, k$ there exists an element $p_{i j}$ in $E(X: Y)$ such that $p_{i j}\left(x_{1 j}\right)=x_{i j}$. An argument used in a previous paragraph of this proof shows that each $p_{\imath j}$ maps $X_{j}$ onto itself. By Lemma 1.3, no two $p_{i j}$ 's agree at any point of $X_{j}$. Upon applying Corollary 1.7 for each $p_{i j}$ and then taking the intersection of all of the open and closed sets in $X$ obtained, there exists an open and closed set $Q_{j}$ in $X$ which contains $X_{j}$ and has the following properties: card $\left(p^{-1}(y) \cap Q_{j}\right)=k$ for all $y \in p\left(Q_{j}\right) ; p_{i j}\left(Q_{j}\right)=Q_{j}$ for each $i=1,2, \cdots, k$; and $p^{-1}(D) \cap Q_{j}=X_{j}$. For any $x$ in $Q_{j}$; with $p(x)=y,\left\{p_{i j}(x): i=1,2, \cdots, k\right\}=p^{-1}(y) \cap Q_{j}$. Consequently, no two $p_{i j}$ 's agree at any point of $Q_{j}$.

By replacing each $Q_{j}$ by $Q_{j} \cap\left(\bigcap_{j=1}^{m} p\left(Q_{j}\right)\right)$ if need be, we may and 
do assume that $p$ maps each $Q_{j}$ onto a set $Q_{0}$ in $Y$. Furthermore, we assume the $Q_{j}$ 's are mutually disjoint since we can select $m$ mutually disjoint open and closed sets $K_{1}, \cdots, K_{m}$ in $X$ containing $X_{1}, \cdots, X_{m}$ respectively and then (Corollary 1.7) pick the $Q_{j}$ 's such that in addition to all of the above properties, $X_{j} \subset Q_{j} \subset K_{j}$ for each $j$. It follows from these assumptions that $p^{-1}\left(Q_{0}\right)=\bigcup_{j=1}^{m} Q_{j}$.

The next step in the proof is to show that without the loss of generality, we may assume that for each $j=1,2, \cdots, m, E_{j}=\left\{p_{i j}: i=\right.$ $1,2, \cdots, k\}$ is a subgroup of $E\left(Q_{j}: Q_{0}\right)$. This part of the proof is accomplished in two steps.

( i ) Corresponding to each integer $i(1 \leqq i \leqq k)$, there exists an integer $r(1 \leqq r \leqq k)$ such that $p_{r j}\left(x_{1 j}\right)=p_{i j}^{-1}\left(x_{1 j}\right)$. By Lemma 1.3, $H_{i}=$ $\left\{x \in X: p_{r j}(x)=p_{i j}^{-1}(x)\right\}$ is an open and closed subset of $X$. Thus $X_{j} \subset H_{i}$ for $i=1,2, \cdots, k$. Let $T_{j}=Q_{j} \cap\left(\bigcap_{i=1}^{k} H_{i}\right)$. This set is open and closed in $X, X_{j} \subset T_{j} \subset Q_{j}$ and the elements of $E_{j}$ restricted to $T_{j}$ are closed with respect to the operation of taking inverse mappings. We now modify the $T_{j}$ 's so that $p$ maps each $T_{j}$ onto a subset $T_{0}$ in $Y$ and $p^{-1}\left(T_{0}\right)=\bigcup_{j=1}^{k} T_{j}$. Therefore we may and do assume $T_{j}=Q_{j}$ for $j=1,2, \cdots, k$.

(ii) For any two distinct integers $r$ and $s(1 \leqq r, s \leqq k)$ there exists an integer $q$ such that $p_{q j}\left(x_{1 j}\right)=p_{r j}\left(p_{s j}\left(x_{1 j}\right)\right)$. An application of Lemma 1.3 yields an open and closed set in $X$ which contains $X_{j}$ and on which $p_{q j}=p_{r j} p_{s j}$. We now repeat this argument for each of the $k^{2}$ elements in $E_{j} \times E_{j}$. Upon taking the intersection of all of the sets obtained, we get an open and closed set $O_{j}$ in $X$ which contains $X_{j}$. Set $Z_{j}=Q_{j} \cap O_{j}$. This is an open and closed set such that the elements of $E_{j}$ restricted to $Z_{j}$ are closed with respect to the operation of taking composite mappings. We modify the sets $Z_{j}$ as above. Therefore we may and do assume $Z_{j}=Q_{j}$ for each $j$. We have shown, therefore, that without loss of generality, $E_{j}=\left\{p_{i j}: i=1,2, \cdots, k\right\}$ can be assumed to be a subgroup of $E\left(Q_{j}: Q_{0}\right)$ for each $j=1,2, \cdots, m$. Indeed, $E_{j}$ is a $k^{\text {th }}$ order subgroup which is simply transitive on the fibers of $Q_{j}(j=1,2, \cdots, m)$. (That is, for each $y$ in $Q_{0}$ and any two points $x$ and $x^{\prime}$ in $p^{-1}(y) \cap Q_{j}$, there exists a unique $i$ such that $p_{i j}(x)=x^{\prime}$.) For each $j$, we redefine each $p_{i j}(i=1,2, \cdots, k)$ to be the identity mapping on $X-Q_{j}$. Let $E_{0}=E_{1} \times E_{2} \times \cdots \times E_{m}$. $E_{0}$ is an $n^{t h}$ order subgroup of $E\left(p^{-1}\left(Q_{0}\right): Q_{0}\right)$ which is simply transitive on the fibers of $p^{-1}\left(Q_{0}\right)$. We also view $E_{0}$ as a subgroup of $E(X: Y)$.

To summarize the proof thus far, corresponding to each component $D$ in $Y$, there exists an open and closed set $Q_{0}$ in $Y$ containing $D$ and there also exists an $n^{\text {th }}$ order subgroup $E_{0}$ of $E\left(p^{-1}\left(Q_{0}\right): Q_{0}\right)$ which is simply transitive on the fibers of $p^{-1}\left(Q_{0}\right)$.

Using the compactness of $Y$, we extract a finite covering of $Y$ by open and closed sets $Y_{1}, Y_{2}, \cdots, Y_{s}$ such that corresponding to each 
$Y_{r}$, there exists an $n^{t h}$ order subgroup $E_{r}=\left\{\phi_{1 r}, \phi_{2 r}, \cdots, \phi_{n r}\right\}$ of $E\left(p^{-1}\left(Y_{r}\right): Y_{r}\right)$ which is simply transitive on the fibers of $p^{-1}\left(Y_{r}\right)$. Also, each of the $\phi_{i r}$ 's is the identity mapping off of $p^{-1}\left(Y_{r}\right)$. Finally, we may and do assume the $Y_{r}$ 's are mutually disjoint since if not, set $Y_{1}^{\prime}=Y$, and for $2 \leqq r \leqq s$, set $Y_{r}^{\prime}=Y_{r}-\bigcup_{i=1}^{r-1} Y_{\imath}^{\prime}$.

For each $i=1,2, \cdots, n$, define the mapping $\phi_{i}$ of $X$ into itself by $\phi_{i}\left|p^{-1}\left(Y_{r}\right)=\dot{\phi}_{i r}\right| p^{-1}\left(Y_{r}\right)$ for $r=1,2, \cdots, s$. Since each $\dot{\phi}_{i r}$ maps $p^{-1}\left(Y_{r}\right)$ onto itself and is the identity mapping off of $p^{-1}\left(Y_{r}\right)$, each $\dot{\phi}_{i}$ is a well-defined homeomorphism in $E(X: Y)$. Also the set $\left\{\dot{\phi}_{1}, \phi_{2}, \cdots\right.$, $\left.\phi_{n}\right\}$ has the property that no two of its elements agree at any point of $X$. That is, the set $E=\left\{\phi_{1}, \phi_{2}, \cdots, \phi_{n}\right\}$ is simply transitive on the fibers of $X$. This completes the proof of Lemma 1.10.

We emphasize that the set $E$ given by the above lemma does not in general form a subgroup of $E(X: Y)$. Also, the elements in the set $E$ are not unique. The proof of Lemma 1.10 yields the following corollary, which will be exploited in the final section of this paper.

Corollary 1.11. Assume $(X, p)$ is a covering space of $Y$ and also assume $E(X: Y)$ is transitive on the fibers of $X$. Then there exists a finite covering of $Y$ by mutually disjoint open and closed sets $Y_{1}, Y_{2}, \cdots, Y_{s}$ with the property that for each $r$, there exists an $n^{\text {th }}$ order subgroup $E_{r}$ of $E\left(p^{-1}\left(Y_{r}\right): Y_{r}\right)$ which is simply transitive on the fibers of $p^{-1}\left(Y_{r}\right)$ and each of whose elements is the identity mapping off of $p^{-1}\left(Y_{r}\right)$.

There is one case where the set $\left\{\dot{\phi}_{1}, \cdots, \phi_{n}\right\}$ given by Lemma 1.10 does form a subgroup of $E(X: Y)$.

Corollary 1.12. Assume $(X, p)$ is a covering space of a connected space $Y$. Then $E(X: Y)$ is transitive on the fibers of $X$ if and only if there exists an $n^{\text {th }}$ order subgroup of $E(X: Y)$ which is simply transitive on the fibers of $X$. Also, if $X$ is connected, then $E(X: Y)$ is transitive on the fibers of $X$ if and only if it is simply transitive on the fibers of $X$. (This implies that $E(X: Y)$ is transitive on the fibers of $X$ if and only if its order is $n$ ).

The final topic in this section deals with the "orbit space" of a finite subgroup of $E(X: Y)$. We assume $(X, p)$ is a covering space of $Y$ and let $E$ be a finite subgroup of $E(X: Y)$. Let $R=\left\{\left(x, x^{\prime}\right) \in X \times X\right.$ : for some $\left.\phi \in E, \phi(x)=x^{\prime}\right\} . \quad R$ is a closed subset of $X \times X$ since $R=$ $\bigcup_{\phi \in E}\left\{\left(x, x^{\prime}\right) \in X \times X: \phi(x)=x^{\prime}\right\}$. Denote by $X / E$ the space of equivalence classes determined by the equivalence relation defined by $R$. We endow this space with the quotient topology. That is, the largest topology (greatest number of open sets) which makes the projection map 
$P: X \rightarrow X / E$ continuous. Thus a set $U$ is open in $X / E$ if and only if $P^{-1}(U)$ is an open subset of $X$ (see [10, p. 94] for details). If $A$ is an open subset of $X, R[A]=\left\{x \in X\right.$ : for some $\left.x^{\prime} \in A,\left(x, x^{\prime}\right) \in R\right\}=$ $\left\{x \in X\right.$ : for some $x^{\prime} \in A$ and $\left.\phi \in E, \phi(x)=x^{\prime}\right\}=\bigcup_{\phi \in E} \phi(A)$. Thus $R[A]$ is an open subset of $X$. Therefore, $P$ is an open mapping of $X$ onto $X / E[10$, p. 97]. It follows from [10, p. 98] that $X / E$ is a Hausdorff space. Moreover, since $P$ is a continuous mapping, $X / E$ is a compact space. Consequently, $P$ is also a closed mapping.

To summarize, if $E$ is a finite subgroup of $E(X: Y)$, then the "orbit space" $X / E$ is a compact Hausdorff space with respect to the quotient topology. Also the projection mapping $P$ of $X$ onto $X / E$ is a continuous, open and closed mapping. If follows from Theorem 1.1 that $(X, P)$ is a covering space of $X / E$ if and only if card $\left(P^{-1}(P(x))\right)$ is a finite constant independent of $P(x) \in X / E$. We also note that the subgroup $E$ of $E(X: Y)$ is transitive on the fibers of $(X, P)$.

Lemma 1.13. Assume $(X, p)$ is a covering space of $Y$ and assume $E$ is a finite subgroup of $E(X: Y)$ with the property that no two elements of $E$ agree at any point of $X$. Then $(X, P)$ is a covering space of $X / E$. Conversely, if $(X, P)$ is a covering space of $X / E$, then $E$ possesses a set of $k\left(=\operatorname{card}\left(P^{-1}(P(x))\right)\right.$ for all $\left.P(x) \in X / E\right)$ elements which is simply transitive on the fibers of $(X, P)$.

Proof. From the above remarks, we need only show that card $\left(P^{-1}(P(x))\right)$ is a constant independent of $P(x) \in X / E$. Since $P^{-1}(P(x))=$ $\bigcup_{\phi \in E}\left\{x^{\prime} \in X: \phi(x)=x^{\prime}\right\}$, the assumption on $E$ implies that for each $P(x) \in X / E$, card $\left(P^{-1}(P(x))\right)=$ order of $E$.

Since $E$ is transitive on the fibers of $(X, P)$, the converse follows from Lemma 1.10. This completes the proof.

2. After presenting some basic facts concerning Arens-Hoffman extensions, we give a necessary and sufficient condition for the generating polynomial to factor into monic linear factors over $B$. The final objective of this section is to apply the fundamental theorem of the Galois theory for commutative rings [4].

We assume $A$ is a commutative Banach algebra over the complex field $\mathscr{C}$ and we assume $A$ possesses an identity element $e$. Let $\alpha(x)=$ $x^{n}+\sum_{\imath=0}^{n-1} \alpha_{i} x^{i}$ be a monic polynomial in $A[x]$. Denote by $(\alpha(x))$ the principal ideal in $A[x]$ generated by $\alpha(x)$. Then $\mathrm{R}$. Arens and $\mathrm{K}$. Hoffman have shown in [2] that $B=A[x] /(\alpha(x))$ possesses a family of equivalent norms with respect to which $B$ is a Banach algebra with the property that the natural embedding of $A$ into $B$ is an isometric isomorphism of $A$ onto a closed subalgebra of $B$. The family of norms is given by 


$$
\left\|\sum_{i=0}^{n-1} a_{i} x^{i}+(\alpha(x))\right\|=\sum_{i=0}^{n-1}\left\|a_{i}\right\| t^{i}
$$

where $t$ is any positive number such that $\sum_{i=0}^{n-1}\left\|\alpha_{i}\right\| t^{i} \leqq t^{n}$. Since such a $t$ always exists, we take $t=1$. We refer to $B$ as the ArensHoffman extension of $A$ with respect to $\alpha(x)$.

We denote the coset $x+(\alpha(x))$ in $B$ by $x$ and the coset $a+(\alpha(x))$ in $B$ by $a$ for any $a \in A$. Thus any element in $B$ is uniquely expressible in the form $\sum_{i=0}^{n-1} a_{i} i^{i}$ for $a_{i} \in A$. Also, the norm of such an element is $\left\|\sum_{i=0}^{n-1} a_{i} \chi^{i}\right\|=\sum_{i=0}^{n-1}\left\|a_{i}\right\|$ where the latter norm is the given norm in $A$.

If $\beta(x)=\sum_{i=0}^{m} \beta_{i} x^{i} \in A[x]$, we view $x$ as an indeterminant over $\hat{A}$ ( $=$ Gelfand representation of $A$ ) and $\mathscr{C}$ as well as an indeterminant over $A$ and we let $\hat{\beta}(x)$ and $\beta_{h}(x)$ denote, respectively, the polynomials $\sum_{i=0}^{m} \widehat{\beta}_{i} x^{i}$ in $\hat{A}[x]$ and $\sum_{i=0}^{m} \widehat{\beta}_{i}(h) x_{i}$ in $\mathscr{C}[x]$ for $h \in \Phi_{A}$. Arens and Hoffman have shown that if $B=A[x] /(\alpha(x))$, then $\Phi_{B}$ is identifiable with the set $\left\{(h, \lambda) \in \Phi_{A} \times \mathscr{C}: \alpha_{h}(\lambda)=0\right\}$ together with the relative topology of $\Phi_{A} \times \mathscr{C}\left[2\right.$, Th. 4.2]. (We note that the carrier space $\Phi_{\mathfrak{H}}$ of any commutative Banach algebra $\mathfrak{A}$ over $\mathscr{C}$ with identity is a compact Hausdorff space.) The projection mapping $\pi$ of $\Phi_{B}$ onto $\Phi_{A}$ defined by $\pi(h, \lambda)=h$ for $(h, \lambda) \in \Phi_{B}$ is a continuous open mapping [12, §1].

If $\beta(x) \in A[x]$, the discriminant $d_{\beta}$ of $\beta(x)$ is defined as in $[15, \mathrm{p}$. 82] and is an element of $A$. Furthermore for $h \in \Phi_{A}, \hat{d}_{\beta}(h)$ is the discriminant of $\beta_{h}(x)$. Thus $\hat{d}_{\beta}(h)=0$ if and only if $\beta_{h}(x)=0$ has at least one root of multiplicity $\geqq 2$. Throughout this paper we will be assuming that the generating polynomial $\alpha(x)$ has an invertible discriminant $d$. Thus $\hat{d}(h) \neq 0$ for each $h \in \Phi_{A}$. Therefore, $\pi^{-1}(h)$ consists of precisely $n$ (= degree of $\alpha(x)$ ) distinct points in $\Phi_{B}$ for each $h \in \Phi_{A}$. In order to simplify the statements of our results, we denote by $I(A)$ (or simply $I$ if there is no confusion as to the algebra in question) the collection of all monic polynomials in $A[x]$ having an invertible discriminant in $A$.

For the remainder to this paper we assume $A$ is a commutative Banach algebra over the complex field $\mathscr{C}, A$ has an identity element $e$, and $B=A[x] /(\alpha(x))$ is the Arens-Hoffman extension of $A$ with respect to the $n^{\text {th }}$ degree polynomial $\alpha(x) \in I$.

From the above remarks and from Theorem 1.1, we have the following:

\section{1. $\left(\Phi_{B}, \pi\right)$ is a covering space of $\Phi_{A}$.}

Another proof of this follows from [12, Proposition 1.3].

The following is our criterion for showing that a monic polynomial in $A[x]$ factors into monic linear factors over $B$. 
Lemma 2.2. Let $\gamma(x)$ be an $n^{\text {th }}$ degree monic polynomial in $A[x]$ and let $\mathfrak{A}$ be any Banach algebra extension of $A$. (i.e., $\mathfrak{A}$ is a Banach algebra which possesses a closed subalgebra isomorphic and isometric to $A$.)

If there exists $b_{1}, b_{2}, \cdots, b_{n}$ in $\mathfrak{U}$ such that $\gamma\left(b_{i}\right)=0$ for each $i$, and if $\left(b_{i}-b_{j}\right)^{-1} \in \mathfrak{A}$ for all $i \neq j$, then $\gamma(x)=\prod_{i=1}^{n}\left(x-b_{i}\right)$ in $\mathfrak{A}[x]$.

Proof. Let $\beta(x)=\prod_{i=1}^{n}\left(x-b_{i}\right)$. Since both $\gamma(x)$ and $\beta(x)$ are monic polynomials of the same degree in $\mathfrak{X}[x]$, there exists a polynomial $r(x) \in \mathfrak{A}[x]$ such that $\gamma(x)=\beta(x)+r(x)$ where either $r(x) \equiv 0$ or else the degree of $r(x)$ is less than $n$. We assume $r(x) \not \equiv 0$. Since $r\left(b_{1}\right)=0$, $r(x)=\left(x-b_{1}\right) q_{1}(x)$. Also, since $r\left(b_{2}\right)=\left(b_{2}-b_{1}\right) q_{1}\left(b_{2}\right)=0$ and $\left(b_{2}-b_{1}\right)^{-1} \in \mathfrak{A}$, $q_{1}\left(b_{2}\right)=0$. Thus $r(x)=\left(x-b_{1}\right)\left(x-b_{2}\right) q_{2}(x)$. Continuing in this fashion, $r(x)=\beta(x) q_{n}(x)$. Thus the degree of $r(x)$ is at least $n$. This is a contradiction and the proof is complete.

The next theorem uses the full machinery for covering spaces which was developed in the first section.

THEOREM 2.3. A necessary and sufficient condition for $\alpha(x)$ to factor into monic linear factors over $B$ is that the group $E\left(\Phi_{B}: \Phi_{A}\right)$ is transitive on the fibers of $\Phi_{B}$. Moreover, if the condition does hold, we may select the $n$ distinct roots of $\alpha(x)=0$ so that each of them generates $B$ over $A$.

Proof. Assume $\alpha(x)=\prod_{i=1}^{n}\left(x-b_{i}\right)$, where the $b_{i}$ 's are all distinct elements of $B$. Since $\alpha(x) \in I$, for each $(h, \lambda) \in \Phi_{B}$ and for each pair of distinct integers $i$ and $j, \hat{b}_{i}(h, \lambda) \neq \hat{b}_{j}(h, \lambda)$. For $i=1,2, \cdots, n$, define the mappings $p_{i}$ of $\Phi_{B}$ into itself by $p_{i}(h, \lambda)=\left(h, \hat{b}_{i}(h, \lambda)\right)$ for $(h, \lambda) \in \Phi_{B}$. Each $p_{i}$ is a continuous mapping, which by Lemma 1.2 is also open. (There are examples to show that the $p_{i}$ 's are not necessarily one-to-one.) For any $(h, \lambda) \in \Phi_{B},\left\{\hat{b}_{i}(h, \lambda): i=1,2, \cdots, n\right\}$ consists of the $n$ distinct roots of $\alpha_{h}(x)=0$. It follows that for any $h \in \Phi_{A}$ and $(h, \lambda) \in \pi^{-1}(h), \pi^{-1}(h)=\left\{p_{i}(h, \lambda): i=1,2, \cdots, n\right\}$.

To show $E\left(\Phi_{B}: \Phi_{A}\right)$ is transitive on the fibers of $\Phi_{B}$, let $h \in \Phi_{A}$ and assume $(h, \lambda)$ and $\left(h, \lambda^{\prime}\right)$ are two points of $\pi^{-1}(h)$. From the above paragraph, there exists two integers $j$ and $j^{\prime}$ such that $p_{j}(h, \lambda)=$ $\left(h, \lambda^{\prime}\right)$ and $p_{j},\left(h, \lambda^{\prime}\right)=(h, \lambda)$. By Lemma 1.6, there exists a homeomorphism $\dot{\phi} \in E\left(\Phi_{B}: \Phi_{A}\right)$ such that $\dot{\phi}(h, \lambda)=\left(h, \lambda^{\prime}\right)$. Thus this part of the proof is complete.

Conversely, assume $E\left(\Phi_{B}: \Phi_{A}\right)$ is transitive on the fibers of $\Phi_{B}$. By Lemma 1.10 there exists a set $\left\{\phi_{1}, \phi_{2}, \cdots, \phi_{n}\right\}$ in $E\left(\Phi_{B}: \Phi_{A}\right)$ which 
is simply transitive on the fibers of $\Phi_{B}$. Define, for each $i=1,2, \cdots, n$, the functions $f_{i}$ of $\Phi_{B}$ into itself by $f_{i}(h, \lambda)=\hat{\mathfrak{x}}\left(\phi_{i}(h, \lambda)\right)$ for $(h, \lambda) \in \Phi_{B}$. Since each $f_{i}$ is a simple root of $\alpha_{h}(x)=0$, by the Arens-Calderon Theorem [1, Th. 7.2], there exists elements $b_{1}, \cdots, b_{n}$ in $B$ such that $\alpha\left(b_{i}\right)=0$ and $\hat{b}_{i}=f_{i}$. The fact that each $b_{i}$ seperates each fiber of $\Phi_{B}$ implies by [11, Th. 7.2] that each $b_{i}$ generates $B$ over $A$. Thus $A\left[b_{i}\right]=B$. Now for any two distinct integers $i$ and $j$, and for any $(h, \lambda) \in \Phi_{B},\left(b_{i}-b_{j}\right)^{\wedge}(h, \lambda)=\hat{\mathfrak{x}}\left(\phi_{i}(h, \lambda)\right)-\hat{\mathfrak{x}}\left(\phi_{j}(h, \lambda)\right) \neq 0$ since no two of the $\phi_{j}$ 's agree at any point of $\Phi_{B}$. Therefore $\left(b_{i}-b_{j}\right)^{-1} \in B$. An application of Lemma 2.2 completes the proof.

Both the necessary and the sufficient condition in the above theorem are true if the assumption that $\alpha(x) \in I$ is slightly weakened. Also, the former condition is true if $A$ is a normed algebra. (See [3], for details.)

Denote by $G(B: A)$ the group of all automorphisms $g$ on $B$ such that $g(a)=a$ for all $a \in A$. The following facts enable us to translate a property of $G(B: A)$ into one of $E\left(\Phi_{B}: \Phi_{A}\right)$ and thus utilize the material in the first section of this paper. For any $g \in G(B: A)$, the dual mapping $g^{*}$ of $g$ is defined by $\hat{b}\left(g^{*}(h, \lambda)\right)=g(b)^{\wedge}(h, \lambda)$ for all $(h, \lambda) \in \Phi_{B}$ and $b \in B$. Since $g^{*} \in E\left(\Phi_{B}: \Phi_{A}\right)([11$, Lemma 6.2]), we have a mapping from $G(B: A)$ into $E\left(\Phi_{B}: \Phi_{A}\right)$. This mapping is order reversing and, by [11, Corollary 6.5], it is also one-to-one and onto. Thus $G(B: A)$ is anti-isomorphic to $E\left(\Phi_{B}: \Phi_{A}\right)$. For any subgroup $G$ of $G(B: A)$, we denote by $G^{*}$ the image of $G$ under this anti-isomorphism.

For the remainder of this section, we assume $G(B: A)$ possesses an $n^{\text {th }}$ order subgroup $G_{0}(B: A)$ with the property that $G_{0}(B: A)^{*}=$ $E_{0}\left(\Phi_{B}: \Phi_{A}\right)$ is simply transitive on the fibers of $\Phi_{B}$. Thus, for example, if $\Phi_{A}$ is connected, then by Corollary 1.12 and Theorem 2.3, the existence of $G_{0}(B: A)$ is a necessary and sufficient condition for $\alpha(x)$ to factor into monic linear factors over $B$. If follows from Lemma 2.2 that

2.4. If $G_{0}(B: A)=\left\{g_{1}, g_{2}, \cdots, g_{n}\right\}$, then $\alpha(x)=\prod_{i=1}^{n}\left(x-g_{i}(\mathfrak{x})\right)$.

Lemma 2.5. Let $F_{0}=\left\{b \in B: g(b)=b\right.$ for all $\left.g \in G_{0}(B: A)\right\}$ be the fixed algebra of $G_{0}(B: A)$. Then $F_{0}=A$.

Proof. Assume $G_{0}(B: A)=\left\{g_{1}, \cdots, g_{n}\right\}$ and set $b_{j}=g_{j}($ r. $)$ for $j=$ $1,2, \cdots, n$. If $b=\sum_{\imath=0}^{n-1} a_{i} x^{i}$ is any element in $F_{0}$, then $b$ is a symmetric function of the $b_{j}$ 's. Therefore, $b$ can be written as a polynomial in the elementary symmetric functions of the $b_{j}$ 's with coefficients in $A$. But in view of 2.4, these elementary symmetric functions are the coefficients of $\alpha(x)$. Consequently, $b \in A$. This means $F_{0} \subset A$. The proof is complete since the reverse inclusion always holds. 
Definition. ([4, Definition 1.4 and Th. 1.3, pp. 18-20]). Let $G$ be a finite group of automorphisms on a commutative ring $S$ and let $R=\{s \in S: g(s)=s$ for all $g \in G\}$. Then $S$ is called a Galois extension of $R$ with Galois group $G$ if for any $g \in G$ and any maximal ideal $M$ in $S$, there exists an element $s \in S$ such that $s-g(s) \notin M$.

As is shown in [4], $S$ is a Galois extension of $R$ with Galois group $G$ if and only if $S$ is a separable $R$-algebra (i.e., $S$ is a projective $S \otimes_{R} S$-module) and the elements of $G$ are pairwise strongly distinct. (Two ring homomorphisms $h_{1}$ and $h_{2}$ from $T$ into $U$ (both commutative rings) are called strongly distinct if for any nonzero idempotent $u$ in $U$ there exists an element $t \in T$ such that $h_{1}(t) u \neq h_{2}(t) u$.)

Lemma 2.6. If $G_{0}(B: A)$ is a subgroup of $G(B: A)$ such that $G_{0}(B: A)^{*}$ is simply transitive on the fibers of $\Phi_{B}$, then $B$ is a Galois extension of $A$ with Galois group $G_{0}(B: A)$.

Proof. By Lemma 2.5, $F_{0}=A$. It follows from the fact that $G_{0}(B: A)$ is simply transitive on the fibers of $\Phi_{B}$ that if $g_{1}$ and $g_{2}$ are two distinct automorphisms in $G_{0}(B: A)$, then $\left(g_{1}(\mathfrak{x})-g_{2}\left(x_{0}\right)\right)^{-1} \in B$. Thus for any $g \in G_{0}(B: A)$ not equal to the identity, $(x-g(x))^{-1} \in B$. In particular, $(\mathfrak{x}-g(\mathfrak{x}))$ is not in any maximal ideal of $B$. This completes the proof.

In the event $\Phi_{A}$ is connected, the above result is not new (see [9, Th. 2.2]).

Definition. ([4, p. 22]). Let $\mathfrak{i}$ be any Banach algebra which contains $A$ and which is a Galois extension of $A$ with Galois group $G$. Then a subalgebra $F$ of $\mathfrak{A}$ is called $G$-strong if the restrictions to $F$ of any two elements of $G$ are either equal or strongly distinct as maps from $F$ into $\mathfrak{A}$.

Theorem 2.7. ([4, Th. 2.3, p. 24]). Assume $G_{0}(B: A)$ is a subgroup of $G(B: A)$ such that $G_{0}(B: A)^{*}$ is simply transitive on the fibers of $\Phi_{B}$. Then there exists a one-to-one lattice inverting correspondence between the subgroups of $G_{0}(B: A)$ and the separable $A$-subalgebras of $B$ which are $G_{0}$-strong. If $F$ is a separable A-subalgebra of $B$ which is $G_{0}$-strong, then the corresponding subgroup is $G_{0}(B: F)=$ $\left\{g \in G_{0}(B: A): g(c)=c\right.$ for all $\left.c \in F\right\}$. Moreover, for $g \in G_{0}(B: A), G_{0}(B$, $g(F))=g G_{0}(B: F) g^{-1}$. A subgroup $G$ of $G_{0}(B: A)$ is a normal subgroup if and only if the fixed algebra $F$ of $G$ is mapped onto itself by every element of $G_{0}(B: A)$. In this case, $F$ is a Galois extension of $A$ with Galois group $G_{0}(B: A) / G$. 
3. Our goal in this section is to characterize the $G_{0}$-strong separable $A$-subalgebras of $B$ in terms of our notion of a covering space. This characterization will be established under the assumptions that $A$ is semi-simple and $G(B: A)$ contains an $n^{t h}$ order subgroup $G_{0}(B: A)$ such that $G_{0}(B: A)^{*}$ is simply transitive on the fibers of $\Phi_{B}$. We first present a few facts concerning subalgebras of $B$.

Assume $F$ is a closed subalgebra of $B$ which contains $A$. We define the restriction mapping $V_{F}$ of $\Phi_{B}$ into $\Phi_{F}$ by $V_{F}(h, \lambda)=(h, \lambda) \mid F$ for $(h, \lambda) \in \Phi_{B}$. Since $V_{F}$ is the dual of the injection mapping of $F$ into $B, V_{F}$ is continuous [14, p. 116]. We now show $V_{F}$ maps $\Phi_{B}$ onto $\Phi_{F}$. That is, we show that any maximal ideal $M$ in $F$ extends to a maximal ideal in $B$. By [16, p. 254], $B$ is an integral extension of $A$ (i.e., each element of $B$ is a zero of a monic polynomial in $A[x]$ ). Thus $B$ is an integral extension of $F$. Therefore, by [16, p. 257], each prime ideal in $F$ can be extended to a prime ideal in $B$. Since each maximal ideal in $F$ is a prime ideal, and since each prime ideal in $B$ can be extended to a maximal ideal in $B$, each maximal ideal in $F$ can be extended to a maximal ideal in $B$.

Next, let $\pi_{F}$ denote the restriction mapping of $\Phi_{F}$ onto $\Phi_{A}$ defined by $\pi_{F}(\hbar)=\hbar \mid A$ for each $\hbar \in \Phi_{F}$. Then, as with $V_{F}, \pi_{F}$ is continuous. Any maximal ideal $h^{-1}(0)$ in $A\left(h \in \Phi_{A}\right)$ can be extended to a maximal ideal in $F$ since if $(h, \lambda) \in \pi^{-1}(h)$, then $(h, \lambda)^{-1}(0) \cap F$ is a maximal ideal in $F$. Therefore $\pi_{F}$ maps $\Phi_{F}$ onto $\Phi_{A}$. Finally, $\pi_{F}$ is an open mapping since if $U$ is an open subset of $\Phi_{F}$, then $\pi\left(V_{F}^{-1}(U)\right)$ is an open subset of $\Phi_{A}$. The fact that $\pi_{F} V_{F}=\pi$ implies that $\pi_{F}(U)=\pi\left(V_{F}^{-1}(U)\right)$.

In summary, we have the following:

Proposition 3.1. Assume $F$ is a closed subalgebra of $B$ which contains $A$. Then the restriction mapping $V_{F}$ is a continuous mapping of $\Phi_{B}$ onto $\Phi_{F}$, while the restriction mapping $\pi_{F}$ is an open continuous mapping of $\Phi_{F}$ onto $\Phi_{A}$.

Now let $G$ be a finite subgroup of $G(B: A)$, and let $F=\{b \in B: g(b)=$ $b$ for all $g \in G\}$ be the fixed algebra of $G$. $F$ is a subalgebra of $B$ which contains $A$. Also, since each $g \in G$ is continuous, $F$ is a closed subalgebra. Let $E$ be the subgroup of $E\left(\Phi_{B}: \Phi_{A}\right)$ such that $G^{*}=E$. We denote by $\Phi_{B} / E$ the space of equivalence classes under the equivalence relation defined by means of the group $E$. (Thus $(h, \lambda) \sim\left(h, \lambda^{\prime}\right)$ if and only if for some $\left.\phi \in E, \phi(h, \lambda)=\left(h, \lambda^{\prime}\right)\right)$. Recally from $\S 1$ that $\Phi_{B} / E$ is a compact Hausdorff space and also recall that the projection mapping $P$ is a continuous open mapping of $\Phi_{B}$ onto $\Phi_{B} / E$. We will identify the carrier space $\Phi_{F}$ of $F$.

Theorem 3.2. Let $G$ be a finite subgroup of $G(B: A), F$ the fixed 
algebra of $G$, and $E$ the subgroup of $E\left(\Phi_{B}: \Phi_{A}\right)$ such that $G^{*}=E$. Then there exists a homeomorphism $\gamma$ of $\Phi_{F}$ onto $\Phi_{B} / E$ such that $\gamma V_{F}=P$, where $P$ is the projection mapping of $\Phi_{B}$ onto $\Phi_{B} / E$, and $V_{F}$ is the restriction mapping of $\Phi_{B}$ onto $\Phi_{F}$.

Proof. Define a mapping $\gamma$ of $\Phi_{F}$ onto $\Phi_{B} / E$ by $\gamma\left(V_{F}(h, \lambda)\right)=$ $P(h, \lambda)$ for $(h, \lambda) \in \Phi_{B}$. We must show $\gamma$ is well-defined. Assume $G=\left\{g_{1}, g_{2}, \cdots, g_{r}\right\}$. If, for $h \in \Phi_{A}$ and for any two points $(h, \lambda)$ and $\left(h, \lambda^{\prime}\right)$ in $\pi^{-1}(h), P(h, \lambda) \neq P\left(h, \lambda^{\prime}\right)$, then for each $j=1,2, \cdots, r, g_{j}^{*}(h$, $\lambda) \neq\left(h, \lambda^{\prime}\right)$. By [14, Lemma 2.6.9] there is an element $b \in B$ such that $\hat{b}\left(h, \lambda^{\prime}\right)=0$ and $\hat{b}\left(g_{j}^{*}(h, \lambda)\right)=1$ for each $j$. Let $c=\Pi_{j=1}^{r} g_{j}(b)$. Then $c \in F, \hat{c}\left(h, \lambda^{\prime}\right)=0$ and $\hat{c}(h, \lambda)=\Pi_{j=1}^{r} g_{j}(b)^{\wedge}(h, \lambda)=\Pi_{j=1}^{r} \hat{b}\left(g_{j}^{*}(h, \lambda)\right)=1$. Therefore, $(h, \lambda)\left|F \neq\left(h, \lambda^{\prime}\right)\right| F$. This means $V_{F}(h, \lambda) \neq V_{F}\left(h, \lambda^{\prime}\right)$. Consequently, $\gamma$ is well-defined. It now follows that $\gamma$ is a continuous, one-to-one mapping of $\Phi_{F}$ onto $\Phi_{B} / E$. This completes the proof.

Throughout the remainder of this section we assume $G_{0}(B: A)$ is an $n^{\text {th }}$ order subgroup of $G(B: A)$ such that $G_{0}(B: A)^{*}=E_{0}\left(\Phi_{B}: \Phi_{A}\right)$ is simply transitive on the fibers of $\Phi_{B}$.

Lemma 3.3. Let $F$ be a $G_{0}$-strong separable A-subalgebra of $B$. Then $\left(\Phi_{B}, V_{F}\right)$ is a covering space of $\Phi_{F}$.

Proof. By Theorem 2.7, $F$ is the fixed algebra of $G_{0}(B: F)=$ $\left\{g \in G_{0}(B: A): g(c)=c\right.$ for all $\left.c \in F\right\}$. We let $E_{0}$ be the subgroup of $E_{0}\left(\Phi_{B}: \Phi_{A}\right)$ such that $G_{0}(B: F)^{*}=E_{0}$. By Theorem 3.2, there exists a homeomorphism $\gamma$ of $\Phi_{F}$ onto $\Phi_{B} / E_{0}$ such that $\gamma V_{F}=P$. The fact that $E_{0}$ is a subgroup of $E_{0}\left(\Phi_{B}: \Phi_{A}\right)$ implies that no two elements of $E_{0}$ agree at any point of $\Phi_{B}$. Consequently, by Lemma $1.13,\left(\Phi_{B}, P\right)$ is a covering space of $\Phi_{B} / E_{0}$. It follows that $\left(\Phi_{B}, V_{F}\right)$ is a covering space of $\Phi_{F}$.

Lemma 3.4. Assume $A$ is semi-simple and let $F$ be a closed subalgebra of $B$ which contains $A$. Also suppose $\left(\Phi_{B}, V_{F}\right)$ is a covering space of $\Phi_{F}$. Then $G_{0}(B: F)^{*}=E_{0}\left(\Phi_{B}: V_{F}: \Phi_{F}\right)=\left\{\phi \in E_{0}\left(\Phi_{B}: \Phi_{A}\right): V_{F} \phi=\right.$ $\left.V_{F}\right\}$. Furthermore, if $F$ is $G_{0}$-strong, then the order of $E_{0}\left(\Phi_{B}: V_{F}: \Phi_{F}\right)=$ card $\left(V_{F}^{-1}(\hbar)\right)=k$, and the latter group is simply transitive on the fibers of $\left(\Phi_{B}, V_{F}\right)$.

Proof. Assume $g \in G_{0}(B: F)$. Let $c \in F, \hbar \in \Phi_{F}$ and $(h, \lambda) \in V_{F}^{-1}(\swarrow)$. Then $\widehat{c}(h)=\widehat{c}\left(V_{F}(h, \lambda)\right)=\widehat{c}(h, \lambda)=g(c)^{\wedge}(h, \lambda)=\widehat{c}\left(g^{*}(h, \lambda)\right)=\widehat{c}\left(V_{F}\left(g^{*}(h, \lambda)\right)\right)$. (We use $\hat{c}$ to denote the element $\hat{c} \in \hat{B}$ as well as the element $\hat{c} \in \hat{F}$ ). Since $\hat{F}$ separates the points of $\Phi_{F}, V_{F}(h, \lambda)=V_{F}\left(g^{*}(h, \lambda)\right)$. Therefore $V_{F}=V_{F} g^{*}$. This means $G_{0}(B: F)^{*} \subset E_{0}\left(\Phi_{B}: V_{F}: \Phi_{F}\right)$.

Conversely, if $\phi \in E_{0}\left(\Phi_{B}: V_{F}: \Phi_{F}\right)$, then $V_{F} \phi=V_{F}$. Since $\phi \in E_{0}\left(\Phi_{B}: \Phi_{A}\right)$, 
there exists an automorphism $g \in G_{0}(B: A)$ such that $g^{*}=\phi$. We must show $g \in G_{0}(B: F)$. That is, we must show $g(c)=c$ for all $c \in F$. For any $c \in F$ and $(h, \lambda) \in \Phi_{B}$, we have $g(c)^{\wedge}(h, \lambda)=\hat{c}\left(g^{*}(h, \lambda)\right)=\hat{c}\left(V_{F}(\phi(h\right.$, $\lambda)))=\hat{c}\left(V_{F}(h, \lambda)\right)=\hat{c}(h, \lambda)$. Thus $g(c)^{\wedge}=\hat{c}$ on $\Phi_{B}$. Since $A$ is semisimple and $\alpha(x) \in I, B$ is semi-simple [2, Th. 4.3]. Therefore $g(c)=c$.

To prove the second assertion in the lemma, since $\left(\Phi_{B}, V_{F}\right)$ is a covering space of $\Phi_{F}$ and since no two elements of $E_{0}\left(\Phi_{B}: V_{F}: \Phi_{F}\right)$ agree at any point of $\left(\Phi_{B}, V_{F}\right)$ (i.e., on $\Phi_{B}$ viewed as the covering space $\left(\Phi_{B}, V_{F}\right)$ of $\left.\Phi_{F}\right), k=\operatorname{card}\left(V_{F}^{-1}(\varkappa)\right) \geqq$ order of $E_{0}\left(\Phi_{B}: V_{F}: \Phi_{F}\right)$. We will show that the latter group is transitive on the fibers of $\left(\Phi_{B}, V_{F}\right)$. (This will imply that it is simply transitive on the fibers of $\left(\Phi_{B}, V_{F}\right)$ ).

Let $h \in \Phi_{F}$ and let $(h, \lambda)$ and $\left(h, \lambda^{\prime}\right)$ be any two points in $V_{F}^{-1}(h)$. Since $\pi(h, \lambda)=\pi\left(h, \lambda^{\prime}\right)$, there exists a homeomorphism $\phi$ in $E_{0}\left(\Phi_{B}: \Phi_{A}\right)$ such that $\phi(h, \lambda)=\left(h, \lambda^{\prime}\right)$. Thus $V_{F} \phi(h, \lambda)=V_{F}(h, \lambda)$. By Lemma 1.3, there exists an open and closed set $Q$ in $\Phi_{B}$ such that $V_{F} \phi\left|Q=V_{F}\right| Q$. Let $u$ be the idempotent in $B$ such that $Q=\left\{(h, \lambda) \in \Phi_{B}: \hat{u}(h, \lambda)=1\right\}$. Let $g \in G_{0}(B: A)$ such that $g^{*}=\phi$. We will show that $g \in G_{0}(B: F)$. For any $c \in F$ and any $(h, \lambda) \in Q, \widehat{c}(h, \lambda)=g(c)^{\wedge}(h, \lambda)$. Since $B$ is semisimple, $g(c) u=c u$ for any $c \in F$. Consequently, for any $g_{0} \in G_{0}(B: F)$, $g(c) u=c u=g_{0}(c) u$ for any $c \in F$. The fact that $F$ is $G_{0}$-strong implies that $g$ must be an element of $G_{0}(B: F)$. Therefore $\phi \in E_{0}\left(\Phi_{B}: V_{F}: \Phi_{F}\right)$. Thus the latter group is transitive on the fibers of $\left(\Phi_{B}, V_{F}\right)$. This completes the proof.

Theorem 3.5. Assume $A$ is semi-simple. Let $F$ be a $G_{0}$-strong closed subalgebra of $B$ which contains $A$ and is such that $\left(\Phi_{B}, V_{F}\right)$ is a covering space of $\Phi_{F}$. Then $F$ is the fixed algebra of $G_{0}(B: F)$.

Proof. Let $B_{1}=F[z] /(\alpha(z))$. Since $\alpha(z) \in I(F), B_{1}$ is a semi-simple Banach algebra [2, Th. 4.3] and its carrier space is $\Phi_{B 1}=\left\{(\hbar, \lambda) \in \Phi_{F} \times\right.$ $\left.\mathscr{C}: \alpha_{h}(\lambda)=0\right\}$. Let $\pi_{1}$ be the usual projection of $\Phi_{B 1}$ onto $\Phi_{F}$. Define a mapping $w$ of $\left(\Phi_{B}, V_{F}\right)$ into $\Phi_{B 1}$ by $w(h, \lambda)=\left(V_{F}(h, \lambda), \lambda\right)$ for $(h, \lambda) \in \Phi_{B}$. (Throughout this proof we view $\Phi_{B}$ as the covering space $\left(\Phi_{\bar{B}}, V_{F}\right)$ of $\Phi_{F}$ and will thus write $\left(\Phi_{B}, V_{F}\right)$ in place of $\left.\Phi_{B}\right) . w$ is a continuous, one-to-one mapping of $\left(\Phi_{B}, V_{F}\right)$ onto a compact subset $K$ of $\Phi_{B_{1}}$. Since $w$ is an open mapping (Lemma 1.2), $K$ is an open subset of $\Phi_{B_{1}}$. Furthermore, $\pi_{1}(K)=\pi_{1} w\left(\Phi_{B}\right)=V_{F}\left(\Phi_{B}\right)=\Phi_{F}$.

By [12, Th. 2.1], there exists mutually orthogonal idempotents $u_{1}, u_{2}, \cdots, u_{s}$ in $F$, and polynomials $\beta_{i}(z), Q_{i}(z)$ in $F[z]$ for $i=1,2$, $\cdots, s$ which have the following properties: $(i) e=\sum_{i=1}^{s} u_{i}$, (ii) each $u_{i} \beta_{i}(z)$ is monic in $u_{i} F[z]$, (iii) $K=\bigcup_{i=1}^{s} \Phi_{D_{i}}$ where $D_{i}=u_{i} F[z] /\left(u_{i} \beta_{i}(z)\right)$ and (iv) $\alpha(z)=\sum_{i=1}^{s} u_{i} \beta_{i}(z) Q_{i}(z)$. The fact that $u_{i} \alpha(z) \in I\left(u_{i} F\right)$ implies that $u_{i} \beta_{i}(z) \in I\left(u_{i} F\right)$ for $i=1,2, \cdots, s$. Since card $\left(V_{F}^{-1}(\swarrow)\right)=k$ for each $\hbar \in \Phi_{F}$, and since $w$ is a homeomorphism of $\left(\Phi_{B}, V_{F}\right)$ onto $K$ such 
that $\pi_{1} w=V_{F}, k=\operatorname{card}\left(\pi_{1}^{-1}(h) \cap K\right)$ for each $h \in \Phi_{F}$. It follows from (iii) that the degree of each $u_{i} \beta_{i}(z)$ must be $k$. Let $\beta(z)=\sum_{i=1}^{s} u_{i} \beta_{i}(z)$. This polynomial is monic and of degree $k$ over $F$. Furthermore, since the $u_{i}$ 's are mutually orthogonal, $\beta(z) \in I(F)$.

If we denote the canonical root of $u_{i} \beta_{i}(z)=0$ in $D_{i}$ by $z_{i}$ for $i=1,2, \cdots, s$, then $c=\sum_{i=1}^{s} u_{i} z_{i}$ is a root of $\beta(z)=0$ in $\sum_{i=1}^{s} D_{i}$. Let $D=F[y] /(\beta(y))$. (We change the variable from $z$ to $y$ in order to distinguish between the canonical roots $z$ and $y$ of $\beta(x)=0$ in $B_{1}$ and $D$, respectively). It follows that the mapping $\sum_{i=0}^{k-1} f_{i} \eta^{i} \rightarrow$ $\sum_{j=1}^{s} u_{j} \sum_{i=0}^{k-1} f_{i z_{j}^{i}}=\sum_{i=0}^{k-1} f_{i} c^{i}$ for $f_{i} \in F$ is an isomorphism of $D$ onto $\sum_{\Phi_{j=1}^{s}}^{s} D_{j}$. Therefore $K=\bigcup_{j=1}^{s} \Phi_{D_{j}}$ can be identified topologically with

If $(h, \lambda) \in K$ and if $(h, \lambda) \in V_{F}^{-1}(h)$, then $\lambda=\hat{\mathfrak{x}}(h, \lambda)=\hat{z}\left(V_{F}(h, \lambda)\right.$, $\lambda)=\hat{\mathfrak{y}}(w(h, \lambda))$. This means $\hat{\mathfrak{x}}=\hat{\mathfrak{y}} w$. Thus $\hat{\beta}(\hat{\mathfrak{x}})=0$. This implies, since $B_{1}$ is semi-simple, that $\beta(\mathfrak{r})=0$.

We now show that $B$ (viewed as a Banach algebra extension of $F$ ) can be identified algebraically with $D$. Define the mapping $T$ of $D$ into $B$ by $T\left(\sum_{i=0}^{k=1} f_{i} \eta^{i}\right)=\sum_{i=0}^{k=1} f_{i} x^{i}$ for $f_{i} \in F$. This is a well-defined homomorphism which, since $F[\mathfrak{x}]=B$, maps $D$ onto $B$. If $(h, \lambda) \in\left(\Phi_{B}\right.$, $\left.V_{F}\right)$, then $T(\mathfrak{y})^{\wedge}(h, \lambda)=\hat{\mathfrak{x}}(h, \lambda)=\hat{\mathfrak{y}}(w(h, \lambda))$. Consequently, the dual mapping $T^{*}$ of $T$ must be equal to $w$. By [14, Th. 3.1.17], $T^{*}$ maps $\left(\Phi_{B}, V_{F}\right)$ homeomorphically onto the hull of $T^{-1}(0)$ in $\Phi_{D}$. But $T^{*}=w$ maps $\left(\Phi_{B}, V_{F}\right)$ onto $K=\Phi_{D}$. Thus the hull of $T^{-1}(0)$ in $\Phi_{D}$ must be equal to $\Phi_{D}$. Since $D$ is semi-simple, $T^{-1}(0)=(0)$. Therefore $B$ is isomorphic to $D$.

By (2.4), if $G_{0}(B: A)=\left\{g_{1}, g_{2}, \cdots, g_{n}\right\}$, then $\alpha(x)=\Pi_{i=1}^{n}\left(x-g_{i}(\mathfrak{x})\right) \in B[x]$. For each $i=1,2, \cdots, n$, let $c_{i} \in D$ such that $T\left(c_{i}\right)=g_{i}(\mathfrak{x})$. Then for each $i, \beta\left(c_{i}\right)=\beta\left(T^{-1} g_{i} T(\mathfrak{y})\right)=0$ since $T^{-1} g_{i} T$ is an automorphism on $D$. We relable the $g_{i}$ 's if need be and assume $G_{0}(B: F)=\left\{g_{1}, \cdots, g_{k}\right\}$. (Lemma 3.4 shows that the order of $G_{0}(B: F)$ is indeed $k$.) Since the mapping $g \rightarrow T^{-1} g T$ for $g \in G(B: F)$ is a group isomorphism of $G(B: F)$ onto $G(D: F)$, the latter group possesses a $k^{\text {th }}$ order subgroup $G_{0}(D: F)$ isomorphic to $G_{0}(B: F)$. Since $G_{0}(B: F)^{*}$ is simply transitive on the fibers of $\Phi_{F}$ (Lemma 3.4), and since $w$ is a homeomorphism of $\Phi_{B}$ onto $\Phi_{D}$ such that $\pi_{1} w=V_{F}, G_{0}(D: F)^{*}$ must be simply transitive on the fibers of $\Phi_{D}$. Now, by Lemma $2.5, F$ is the fixed algebra of $G_{0}(D: F)$. Therefore $F$ must also be the fixed algebra of $G_{0}(B: F)$. This completes the proof of Theorem 3.5.

We note that the above theorem need not be true if $A$ is not semi-simple, since there may exist two distinct closed subalgebras of $B$ which contain $A$ and which have the same carrier space.

COROLlary 3.6. Under the same assumptions as in Theorem 3.5, there exists a polynomial $\beta(y) \in I(F)$ of degree $k=\operatorname{card}\left(V_{F}^{-1}(k)\right)$ with 
the property that $B$ (viewed as an extension of $F$ ) can be algebraically and topologically identified with $F[y] /(\beta(y))$.

Proof. From the above proof, the mapping $T$ defined by $T\left(\sum_{i=0}^{k-1} f_{i} \eta^{i}\right)=\sum_{i=0}^{k-1} f_{i} x^{i}$ for $f_{i} \in F$ is an isomorphism of $D$ onto $B$. We show that $T$ is also a homeomorphism.

Assume the norm on $D$ is given by $\left\|\sum_{i=0}^{k-1} f_{i} \eta^{i}\right\|=\sum_{i=0}^{k-1}\left\|f_{i}\right\| t^{i}$ for some positive number $t$. Let $L=\min \left\{t^{i}: i=0,1, \cdots, k-1\right\}$. Then

$$
\begin{aligned}
& \left\|T\left(\sum_{i=0}^{k-1} f_{i} \eta^{i}\right)\right\|=\left\|\sum_{i=0}^{k-1} f_{i} i_{i}^{i}\right\| \leqq \sum_{i=0}^{k-1}\left\|f_{i}\right\| \\
& \leqq L^{-1} \sum_{i=0}^{k-1}\left\|f_{i}\right\| t^{i}=L^{-1}\left\|\sum_{i=0}^{k-1} f_{i} y^{i}\right\| \text {. }
\end{aligned}
$$

Thus $T$ is a continuous mapping of $D$ onto $B$. By the inverse mapping theorem, $T^{-1}$ is a continuous mapping of $B$ onto $D$. This completes the proof.

Combining Lemma 3.3 with Theorem 3.5, we have the following characterization of the fixed algebras of subgroup of $G_{0}(B: A)$.

Theorem 3.7. Assume $A$ is semi-simple, $B$ is the Arens-Hoffman extension of $A$ with respect to the $n^{\text {th }}$ degree polynomial $\alpha(x) \in I$, and $G(B: A)$ contains an $n^{\text {th }}$ order subgroup $G_{0}(B: A)$ such that $G_{0}(B: A)^{*}$ is simply transitive on the fibers $\Phi_{B}$. Then a $G_{0}$-strong closed subalgebra $F$ of $B$ which contains $A$ is the fixed algebra of a subgroup $G$ of $G_{0}(B: A)$ if and only if $\left(\Phi_{B}, V_{F}\right)$ is a covering space of $\Phi_{F}$.

4. In this section, we will prove a theorem similar to Theorem 3.7, but the condition that a subgroup of $E\left(\Phi_{B}: \Phi_{A}\right)$ exists which is simply transitive on the fibers of $\Phi_{B}$ will be weakened.

Our assumptions throughout this portion of the paper are as follows: $A$ is semi-simple, and $\alpha(x)$ factors into monic linear factors over $B$. (Or equivalently by Theorem $2.3, E\left(\Phi_{B}: \Phi_{A}\right)$ is transitive on the fibers of $\Phi_{B}$ ).

By Corollary 1.11, there exists a finite covering of $\Phi_{A}$ by mutually disjoint open and closed sets $Q_{1}, Q_{2}, \cdots, Q_{s}$ with the property that corresponding to each $Q_{j}$, there exists an $n^{\text {th }}$ order subgroup $E_{j}$ of $E\left(\pi^{-1}\left(Q_{j}\right): Q_{j}\right)$ which is simply transitive on the fibers of $\pi^{-1}\left(Q_{j}\right)$. Furthermore, each homeomorphism in $E_{j}$ is the identity homeomorphism off of $\pi^{-1}\left(Q_{j}\right)$. We denote by $E_{0}\left(\Phi_{B}: \Phi_{A}\right)$ the subgroup $E_{1} \times E_{2} \times \cdots \times E_{s}$ of $E\left(\Phi_{B}: \Phi_{A}\right)$. The order of this subgroup is $n \cdot s$.

For each $j$, let $u_{j}$ be the idempotent in $A$ corresponding to the open and closed set $Q_{j}$ in $\Phi_{A}$. (i.e., $Q_{j}=\left\{h \in \Phi_{A}: \widehat{u}_{j}(h)=1\right\}$.) Then $\Phi_{u_{j} A}=Q_{j}$ and $\Phi_{u_{j} B}=\pi^{-1}\left(Q_{j}\right)$ for each $j$. We note that $e=\sum_{j=1}^{s} u_{j}$. For $j=1,2, \cdots, s$, let $G_{j}$ be the $n^{\text {th }}$ order subgroup of $G\left(u_{j} B: u_{j} A\right)$ such 
that $G_{j}{ }^{*}=E_{j}$. We denote by $G_{0}(B: A)$ the subgroup $G_{1} \times G_{2} \times \cdots \times G_{s}$ of $G(B: A)$. It follows that the order of $G_{0}(B: A)$ is $n \cdot s$ and $G_{0}(B: A)^{*}=$ $E_{0}\left(\Phi_{B}: \Phi_{A}\right)$. (In the event $s=1$, then these two subgroups coincide with the subgroups $G_{0}(B: A)$ and $E_{0}\left(\Phi_{B}: \Phi_{A}\right)$ used in $\S 2$ and $\left.\S 3\right)$. If $g \in G_{0}(B: A)$, then $g=\left(g_{1}, g_{2}, \cdots, g_{s}\right)$ where $g_{j} \in G_{j}$ for each $j$. If $b=\sum_{j=1}^{s} u_{j} b \in B$, then $u_{j} g(b)=g\left(u_{j} b\right)=g_{j}\left(u_{j} b\right)$. Also, $g(b)=\sum_{j=1}^{s} g\left(u_{j} b\right)=$ $\sum_{j=1}^{s} u_{j} g\left(u_{j} b\right)=\sum_{j=1}^{s} g_{j}\left(u_{j} b\right)$. We relate fixed algebras of subgroups of $G_{0}(B: A)$ to those of subgroups of $G_{j}$.

4.1. If $F$ is the fixed algebra of a subgroup $G^{\prime}$ of $G_{0}(B: A)$, then for each $j, u_{j} F$ is the fixed algebra of $G_{j}^{\prime}=G^{\prime} \cap G_{j}$.

Proof. If $c \in F$, then since $g_{j}\left(u_{j} c\right)=u_{j} g(c)=u_{j} c$ for each $g_{j} \in G_{j}^{\prime}$, $u_{j} F$ is contained in the fixed algebra of $G_{j}^{\prime}$. Conversely if, for some $c \in B, g_{j}\left(u_{j} c\right)=u_{j} c$ for each $g_{j} \in G_{j}^{\prime}$, then $g\left(u_{j} c\right)=g_{j}\left(u_{j} c\right)=u_{j} c$ for each $g \in G^{\prime}$. Therefore $u_{j} F$ must be the fixed algebra of $G_{j}^{\prime}$.

4.2. If for each $j, F_{j}$ is a subalgebra of $u_{j} B$ which is the fixed algebra of a subgroup $G_{j}^{\prime}$ of $G_{j}$, then $F=\sum_{j=1}^{s} u_{j} F_{j}$ is the fixed algebra of $G^{\prime}=G_{1}^{\prime} \times G_{2}^{\prime} \times \cdots \times G_{s}^{\prime}$.

Proof. For $c \in F, u_{j} c \in F_{j}$ for each $j$. Therefore, for any automorphism $g \in G^{\prime}$, we have $g(c)=\sum_{j=1}^{s} g\left(u_{j} c\right)=\sum_{j=1}^{s} g_{j}\left(u_{j} c\right)=\sum_{j=1}^{s} u_{j} c=c$. This means $F$ is contained in the fixed algebra of $G^{\prime}$. On the other hand, if $g(c)=c$ for some $c \in B$ and for all $g \in G^{\prime}$, then $g_{j}\left(u_{j} c\right)=u_{j} g(c)=$ $u_{j} c$. Therefore $u_{j} c \in u_{j} F_{j}$. Thus $c \in F$. Consequently, $F$ is the fixed algebra of $G^{\prime}$.

As a consequence of Lemma 2.5 and 4.2 , we have

4.3. $A$ is the fixed algebra of $G_{0}(B: A)$.

We also note that by Lemma $2.6, u_{j} B$ is a Galois extension of $u_{j} A$ with Galois group $G_{j}$.

LEMmA 4.4. If $F$ is the fixed algebra of

$$
G_{0}(B: F)=\left\{g \in G_{0}(B: A): g(c)=c\right.
$$

for all $c \in F\}$, then $\left(\Phi_{B}, V_{F}\right)$ is a local covering space of $\Phi_{F}$.

Proof. By 4.1, $u_{j} F$ is the fixed algebra of

$$
G_{j}(B: F)=\left\{g \in G_{j}: g\left(u_{j} c\right)=u_{j} c \text { for all } c \in F\right\} \text {. }
$$

Thus, (Theorem 2.7), $u_{j} F$ is a separable $u_{j} A$-subalgebra of $u_{j} B$ which is $G_{j}$-strong. Now by Lemma 3.3, $\left(\Phi_{u_{j} B}, V_{j}\right)$ is a covering space of 
$\Phi_{u_{j} F}$, where $V_{j}=V_{F} \mid \Phi_{u_{j} B}$. The proof is completed by applying Corollary 1.8.

Lemma 4.5. If $F$ is a closed subalgebra of $B$ which contains $A$ and each $u_{j} F$ is $G_{j}$-strong, and if $\left(\Phi_{B}, V_{F}\right)$ is a local covering space of $\Phi_{F}$, then $F$ is the fixed algebra of $G_{0}(B: F)$.

Proof. By Corollary 1.8, there exists a finite covering of $\Phi_{F}$ by mutually disjoint, open and closed sets $R_{1}, R_{2}, \cdots, R_{t}$ such that for each $i,\left(V_{F}^{-1}\left(R_{i}\right), V_{F} \mid V_{F}^{-1}\left(R_{i}\right)\right)$ is a covering space of $R_{i}$. Let $Z_{i j}=$ $R_{i} \cap \Phi_{u_{j} F}$ for $\mathrm{i}=1,2, \cdots, t$ and $j=1,2, \cdots, s$. Without loss of generality we assume that these sets are mutually disjoint. Let $X_{i j}=$ $V_{F}^{-1}\left(Z_{i j}\right)$ and $Y_{i j}=\pi\left(X_{i j}\right)=V_{F}\left(Z_{i j}\right)$. These open and closed sets are such that $\left(X_{i j}, V_{i j}\right)$ is a covering space of $Z_{i j}$, where $V_{i j}=V_{F} \mid X_{i j}$, and $\left(X_{i j}, \pi_{i j}\right)$ is a covering space of $Y_{i j}$ where $\pi_{i j}=\pi \mid X_{i j}$. Since $Y_{i j} \subset \Phi_{u_{j A}}$ and $E_{j}$ is an $n^{\text {th }}$ order subgroup of $E\left(u_{\jmath} B: u_{j} A\right)$ which is simply transitive of the fibers of $\Phi_{u_{j} B}$, it follows that $E_{i j}=E_{j} \mid X_{i j}$ is an $n^{\text {th }}$ order subgroup of $E\left(X_{i j}: Y_{i j}\right)$ which is simply transitive on the fibers of $X_{i j}$.

For each $i$ and $j$, let $e_{i j}$ be the idempotent in $A$ corresponding to the open and closed set $Y_{i j}$. Also, let $G_{i j}$ be the $n^{\text {th }}$ order subgroup of $G_{j} \mid e_{i j} B$ such that $G_{i j}^{*}=E_{i j}$. It follows that $G_{i j}=G_{j} \mid e_{i j} B$ and thus $G_{j}=G_{1 j} \times G_{2 j} \times \cdots \times G_{t j}$. Since $e_{i j} A$ is the fixed algebra of $G_{i j}$ (Lemma 2.5), by Lemma 2.6, $e_{i j} B$ is a Galois extension of $e_{i j} A$ with Galois group $G_{i j}$.

We next show that each $e_{i j} F$ is $G_{i j}$-strong. By our assumptions on the $u_{j} F$ 's, for each $j$, for any two distinct automorphisms $g_{1}$ and $g_{2}$ in $G_{j}$, and for any idempotent $u_{0}$ in $u_{j} B$, there exists an element $c$ in $F$ such that $g_{1}\left(u_{j} c\right) u_{0} \neq g_{2}\left(u_{j} c\right) u_{0}$. In particular, for $g_{1}, g_{2}$ in $G_{i j}$ and for any idempotent $e_{0}$ in $e_{i j} B \subset u_{j} B$, there exists an element $c \in F$ such that

$$
g_{1}\left(e_{i j} c\right) e_{0}=g_{1}\left(u_{j} c\right) e_{i j} e_{0} \neq g_{2}\left(u_{j} c\right) e_{i j} e_{0}=g_{2}\left(e_{i j} c\right) e_{0} .
$$

Therefore each $e_{i j} F$ is $G_{i j}$-strong.

By Theorem 3.5, $e_{i j} F$ is the fixed algebra of $G_{i j}(B: F)=\left\{g \in G_{i j}\right.$ : $g\left(e_{i j} c\right)=e_{i j} c$ for all $\left.c \in F\right\}$ for each $i$ and $j$. Since the proof of 4.2 does not depend upon the particular decomposition of $F, F$ is the fixed algebra of

$$
\begin{aligned}
& G_{11}(B: F) \times \cdots \times G_{1 s}(B: F) \times \cdots \times G_{t 1}(B: F) \times \cdots \times G_{t s}(B: F) \\
& \quad=G_{1}(B: F) \times \cdots G_{s}(B: F)=G_{0}(B: F) .
\end{aligned}
$$

This completes the proof. 
In conclusion, we have the following charcterization of the fixed algebras of $G_{0}(B: A)$.

Theorem 4.6. Assume $A$ is semi-simple, $B=A[x] /(\alpha(x))$ and $\alpha(x) \in I$ factors into monic linear factors over $B$. Let $G_{0}(B: A)=$ $G_{1} \times G_{2} \times \cdots \times G_{s}$ be defined as in the opening remarks of this section. Then a closed subalgebra $F$ of $B$ which contains $A$ with the property that $u_{j} F$ is $G_{j}$-strong for each $j$ is the fixed algebra of a subgroup of $G_{0}(B: A)$ if and only if $\left(\Phi_{B}, V_{F}\right)$ is a local covering space of $\Phi_{F}$.

The author would like to express his appreciation to Professor John A. Lindberg, Jr. The author would also like to thank the referees for their helpful suggestions and especially for pointing out the error in the original version of Lemma 1.10 .

The author is also indebted to Mr. Philip Downum for a careful reading of the preprint and for pointing out two minor mistakes, which have been corrected.

\section{BIBLIOGRAPHY}

1. R. Arens and A. P. Calderon, Analytic functions of several Banach algebra elements, Ann. of Math. 6 (1955), 204-216.

2. R. Arens and K. Hoffman, Algebraic extensions of normed algebras, Proc. Amer. Math. Soc. 7 (1956), 203-210.

3. D. T. Brown, On some aspects of the Arens-Hoffman extension of Banach algebras, $\mathrm{Ph}$. D. Dissertation, Syracuse University, Syracuse, New York, 1965.

4. S. U. Chase, D. K. Harrison and A. Rosenberg, Galois theory and cohomology of commutative rings, Memoirs, Amer. Math. Soc. No. 52, 1965.

5. C. Chevalley, Theory of Lie groups I, Princeton University Press, Princeton, New Jersey, 1946.

6. G. A. Heuer, Algebraic extensions of Banach algebras, Ph. D. Thesis, Univ. of Minnesota, Minneapolis, Minnesota, 1958.

7. G. A. Heuer and J. A. Lindberg, Algebraic extensions of continuous function algebras, Proc. Amer. Math. Soc. 14 (1963), 337-342.

8. J. Hocking and G. Young, Topology, Addison-Wesley, Reading, Mass., 1961.

9. G. J. Janusz, Separable algebras over commutative rings, Trans. Amer. Math. Soc. 112 (1966), 461-479.

10. J. L. Kelley, General topology, D. Van Nostrand, New York, 1955.

11. J.A. Lindberg, Algebraic extensions of commutative Banach algebras, Pacific J. Math. 14 (1964), 559-584.

12. - Factorization of polynomials over Banach algebras, Trans. Amer. Math. Soc. 112 (1964), 359-368.

13. S. Lubkin, Theory of covering spaces, Trans. Amer. Math. Soc. 104 (1962), 205238.

14. C. E. Rickart, General theory of Banach algebras, D. Van Nostrand, New York, 1960. 
15. B. L. Van der Waerden, Modern Algebra, vol. 1, $2^{\text {nd }}$ Edition, Translated by F. Blum, Frederick Ungar, New York, 1953.

16. O. Zariski and P. Samuel, Commutative algebra, vol. 1, D. Van Nostrand, 1958.

Received April 5, 1967 and in revised form December 12, 1968. This paper is an outgrowth of the author's doctoral dissertation written at Syracuse University under the direction of Professor John A. Lindberg, Jr. While at Syracuse, the author was partially supported by a grant from the U.S. Army Research office in Durham and by a National Aeronautics and Space Administrative Fellowship. The writing of the original version of this paper was supported in part by a Faculty Summer Research grant from Hiram College.

Hiram COLLEGE

HIRAM, OHIO

UNIVERSITY OF PItTSBURGH

Pittsburgh, Pennsylvania 


\section{PACIFIC JOURNAL OF MATHEMATICS}

\section{EDITORS}

H. ROYDEN

Stanford University

Stanford, California

\section{RichaRd PIERCE}

University of Washington Seattle, Washington 98105
J. DugundJI

Department of Mathematics

University of Southern California

Los Angeles, California 90007

BASIL GORDON

University of California

Los Angeles, California 90024

\section{ASSOCIATE EDITORS}
E. F. BECKENBACH
B. H. NeUMANN
F. WOLF
K. YOSHIDA

\section{SUPPORTING INSTITUTIONS}

UNIVERSITY OF BRITISH COLUMBIA CALIFORNIA INSTITUTE OF TECHNOLOGY

UNIVERSITY OF CALIFORNIA

MONTANA STATE UNIVERSITY

UNIVERSITY OF NEVADA

NEW MEXICO STATE UNIVERSITY

OREGON STATE UNIVERSITY

UNIVERSITY OF OREGON

OSAKA UNIVERSITY

UNIVERSITY OF SOUTHERN CALIFORNIA

\author{
STANFORD UNIVERSITY \\ UNIVERSITY OF TOKYO \\ UNIVERSITY OF UTAH \\ WASHINGTON STATE UNIVERSITY \\ UNIVERSITY OF WASHINGTON \\ AMERICAN MATHEMATICAL SOCIETY \\ CHEVRON RESEARCH CORPORATION \\ TRW SYSTEMS \\ NAVAL WEAPONS CENTER
}

The Supporting Institutions listed above contribute to the cost of publication of this Journal, but they are not owners or publishers and have no responsibility for its content or policies.

Mathematical papers intended for publication in the Pacific Journal of Mathematics should be in typed form or offset-reproduced, double spaced with large margins. Underline Greek letters in red, German in green, and script in blue. The first paragraph or two must be capable of being used separately as a synopsis of the entire paper. It should not contain references to the bibliography. Manuscripts, in duplicate if possible, may be sent to any one of the four editors. Please classify according to the scheme of Math. Rev. 36, 1539-1546. All other communications to the editors should be addressed to the managing editor, Richard Arens, University of California, Los Angeles, California, 90024.

50 reprints are provided free for each article; additional copies may be obtained at cost in multiples of 50 .

The Pacific Journal of Mathematics is published monthly. Effective with Volume 16 the price per volume (3 numbers) is $\$ 8.00$; single issues, $\$ 3.00$. Special price for current issues to individual faculty members of supporting institutions and to individual members of the American Mathematical Society: $\$ 4.00$ per volume; single issues $\$ 1.50$. Back numbers are available.

Subscriptions, orders for back numbers, and changes of address should be sent to Pacific Journal of Mathematics, 103 Highland Boulevard, Berkeley, California, 94708.

PUBLISHED BY PACIFIC JOURNAL OF MATHEMATICS, A NON-PROFIT CORPORATION

Printed at Kokusai Bunken Insatsusha (International Academic Printing Co., Ltd.), 7-17. Fujimi 2-chome, Chiyoda-ku, Tokyo, Japan. 


\section{Pacific Journal of Mathematics}

\section{Vol. 30, No. $3 \quad$ November, 1969}

Willard Ellis Baxter, Topological rings with property $(Y) \ldots \ldots \ldots \ldots . \ldots 5$

Sterling K. Berberian, Note on some spectral inequalities of $C . R$.

Putnam ..................................... 573

David Theodore Brown, Galois theory for Banach algebras . ........... 577

Dennis K. Burke and R. A. Stoltenberg, A note on p-spaces and Moore spaces ........................................ 601

Rafael Van Severen Chacon and Stephen Allan McGrath, Estimates of positive contractions....................................... 609

Rene Felix Dennemeyer, Conjugate surfaces for multiple integral problems in the calculus of variations ........................... 621

Edwin O. Elliott, Measures on countable product spaces.............. 639

John Moss Grover, Covering groups of groups of Lie type .............. 645

Charles Lemuel Hagopian, Concerning semi-local-connectedness and cutting in nonlocally connected continua .................. 657

Velmer B. Headley, A monotonicity principle for eigenvalues ........... 663

John Joseph Hutchinson, Intrinsic extensions of rings . . . . . . . . . . . . . 669

Harold H. Johnson, Determination of hyperbolicity by partial

prolongations .................................. 679

Tilla Weinstein, Holomorphic quadratic differentials on surfaces in $E^{3} \ldots 697$

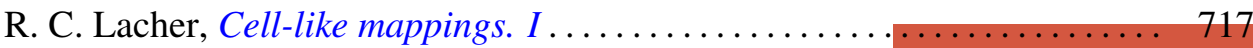

Roger McCann, A classification of centers

Curtis L. Outlaw, Mean value iteration of nonexpansive mappings in a

Banach space...

Allan C. Peterson, Distribution of zeros of solutions of a fourth order

differential equation.

Bhalchandra B. Phadke, Polyhedron inequality and strict convexity .. 765 Jack Wyndall Rogers Jr., On universal tree-like continua .

Edgar Andrews Rutter, Two characterizations of quasi-Frobenius rings

G. Sankaranarayanan and C. Suyambulingom, Some renewal theorems concerning a sequence of correlated random variables...

Joel E. Schneider, A note on the theory of primes........ . .

Richard Peter Stanley, Zero square rings .................

Edward D. Tymchatyn, The 2-cell as a partially ordered space

Craig A. Wood, On general Z.P.I.-rings................ 